\title{
Device physics of organic light-emitting diodes based on molecular materials
}

\author{
Wolfgang Brütting *, Stefan Berleb, and Anton G. Mückl, \\ Experimental Physics II, University of Bayreuth, \\ 95440 Bayreuth, Germany
}

Electrical transport in single- and hetero-layer organic light-emitting diodes (OLEDs) based on aromatic amines like TPD (N,N'-diphenyl-N,N'-bis(3-methylphenyl)-1,1'biphenyl-4,4'-diamine) or NPB (N,N'-diphenyl-N,N'-bis(1-naphthyl)-1,1'-biphenyl-4,4'diamine) and the aluminium chelate complex Alq (tris(8-hydroxyquinolato)aluminium) has been investigated as a function of temperature and organic layer thickness. It is shown that the thickness dependence of the current-voltage $(I-V)$ characteristics provides a unique criterion to discriminate between (1) injection limited behaviour, (2) trap-charge limited conduction with an exponential trap distribution and a field-independent mobility, and (3) trap-free space-charge limited conduction (SCLC) with a field and temperature dependent mobility.

The $I-V$ characteristics of NPB-based hole-only devices with indium-tin oxide anodes are neither purely injection nor purely space-charge limited, although the current shows a square-law dependence on the applied voltage. In Al/Alq/Ca electron-only devices with Alq thickness in the range 100 to $350 \mathrm{~nm}$ the observed thickness and temperature dependent $I-V$ characteristics can be described by SCLC with a hopping-type charge carrier mobility. Additionally, trapping in energetically distributed trap states is involved at low voltages and for thick layers. The electric field and temperature dependence of the charge carrier mobility in Alq has been independently determined from transient electroluminescence. The obtained values of the electron mobility are consistent with temperature dependent $I-V$ characteristics and can be described by both the phenomenological PooleFrenkel model with a zero-field activation energy $\Delta E=0.4-0.5 \mathrm{eV}$ and the Gaussian disorder model with a disorder parameter $\sigma=100 \mathrm{meV}$. Measurements of the bias-dependent capacitance in NPB/Alq hetero-layer devices give clear evidence for the presence of negative charges with a density of about $6.8 \times 10^{11} \mathrm{~cm}^{-2}$ at the organic-organic interface under large reverse bias. This leads to a non-uniform electric field distribution in the hetero-layer device, which has to be considered in device description.

*e-mail: wolfgang.bruetting@uni-bayreuth.de 


\section{INTRODUCTION}

Electroluminescence (EL), that is the generation of light (other than black-body radiation) from condensed matter by electrical excitation, has been investigated in organic molecular solids since the 1950s [1]. Especially the work of Pope et al. and Helfrich et al. $[2,3]$ on single crystals of anthracene in the early 1960s initiated considerable efforts to achieve light-emitting devices from molecular crystals. In spite of the principal demonstration of an operating organic electroluminescent display incorporating even an encapsulation scheme similar to the ones used in nowadays commercial display applications [4], there were several draw-backs preventing practical use of these early devices. For example, neither high enough current densities and light output nor sufficient stability could be achieved. The main obstacles were the high operating voltage as a consequence of the crystal thickness in the micrometer range together with the difficulties in reproducible crystal growth as well as preparing stable and sufficiently wellinjecting contacts to them. Nevertheless, these investigations have established the basic processes involved in organic injection-type EL, namely injection, transport, capture and radiative recombination of oppositely charged carriers inside the organic material (for a review see e.g. $[5,6]$ ).

A further step towards applicable organic electroluminescent devices was made in the 1970s by the usage of thin organic films prepared by vacuum vapor deposition or the Langmuir-Blodgett technique instead of single crystals [7-9]. The reduction of the organic layer thickness well below $1 \mu \mathrm{m}$ allowed to achieve electric fields comparable to those which were applied to single crystals but now at considerably lower voltage. Apart from the morphological instability of these polycrystalline films there arose the problem of fabricating pin-holefree thin films from these materials. These problems could be overcome in the early 1980s by the usage of morphologically stable amorphous films, as demonstrated e.g. by Partridge's work on films of polyvinylcarbazole doped with fluorescent dye molecules [10].

The development of organic multi-layer structures considerably improved the efficiency of lightemission by achieving a better balance of the number of charge carriers of opposite sign and further lowered the operating voltage by reducing the mismatch of energy levels between the organic materials and the electrodes. The consequence of this development was the demonstration of organic lightemitting devices (OLEDs) by Tang et al. with the true potential for lighting and display applications $[11,12]$. By using a hetero-layer structure of a hole conducting aromatic amine and an electron conducting aluminium chelate complex, each a few ten nanometers thick, sandwiched between indiumtin oxide (ITO) and Mg:Ag-alloy electrodes, they could achieve astonishingly high light output, efficiency and life-time at low operating voltage. This breakthrough initiated great efforts in the development of new molecular materials and device structures, especially in Japan [13-15]. Since the end of the 1990s OLEDs have entered the stage of commercialization [16] and are considered as promising candidates for the next generation of large area flat-panel displays $[17,18]$. In addition, since the discovery of EL in conjugated polymers by the Cambridge group in 1990 [19] these materials have also been widely examined and are going to be commercialized with equally good prospects for display and lighting applications as the lowmolecular weight materials $[20,21]$.

It was shown already in the early work on molecular crystals that the relevant mechanism of EL in organic solids is recombination luminescence of injected carriers. As mentioned above, a very successful approach to separately optimize the individual steps involved in organic EL is the concept of multi-layer light-emitting devices using interfaces between different organic materials. The simplest OLED of this kind, which is still widely used for light-emission in the spectral range from the green to the red, incorporates the interface between a hole conducting material (usually a triphenyl-amine derivative) and an electron conducting aluminium chelate complex (Alq), where light-emission is generated in the Alq layer close to the organic-organic interface [11]. (For colour tuning and to achieve higher efficiency and lifetime the Alq layer is frequently doped with fluorescent dyes [12]). The optimization of the yield in these devices requires high and equal densities of positive and negative carriers at the interface. The achievement of this goal necessitates detailed knowledge of the injection process at the electrodes, of the parameters controlling transport through the organic layers and of the energetics at the interfaces.

The organization of this paper is as follows. We will first give a brief survey of device physics of organic light-emitting diodes with the emphasis on the electrical behaviour, especially charge carrier injection and transport. Then we will describe our experimental techniques for device preparation and characterization, and present our experimental results. We will separately discuss current-voltage characteristics of single and hetero-layer devices, extract data on the field and temperature dependent charge carrier mobilities and discuss the electric field distribution in hetero-layer devices.

\section{BASIC CONCEPTS}

\section{A. Electroluminescence in organic solids}

Although it is common to use many concepts derived from inorganic semiconductor physics, one should be aware of the peculiarities and differ- 
ences of organic semiconductors to their inorganic counterparts. Additionally, since most polymeric or low-molecular weight materials used in OLEDs form disordered amorphous films without a macroscopic crystal lattice, it is also not possible to simply adopt mechanisms developed for molecular crystals. Because of the absence of extended delocalized states, charge transport is usually not a coherent motion in well-defined bands but rather a stochastic process of hopping between localized states, which leads to the typically observed low carrier mobilities $\left(\mu \ll 1 \mathrm{~cm}^{2} / \mathrm{Vs}\right)$. Consequently, excitations are localized on either individual molecules or a few monomeric units only of a polymer chain and usually have a large exciton binding energy of some tenths of an eV. Additionally, many of the materials in OLEDs are wide-gap materials with energy gaps of 2 to $3 \mathrm{eV}$, sometimes even more. Therefore the intrinsic concentration of thermally generated free carriers is generally negligible (less than $10^{10} \mathrm{~cm}^{-3}$ ) and from this viewpoint the materials can be considered more as insulators than as semiconductors. Unlike in inorganic semiconductors, impurities usually act as traps rather than as sources of extrinsic mobile charge carriers. There are exceptions, however, one example being the polymer polyphenylenevinylene (PPV) prepared by a precursor route, where doping by a chemical reaction with ITO substrates has been found to be responsible for the observed Schottky diode behaviour [22-25]. Controlled doping, mostly by chemical or electrochemical means, has been studied extensively in polymeric semiconductors [26], but not yet to a large extend in low-molecular weight materials $[27,28]$. Sources of traps in organic semiconductors can be residual impurities from the synthesis of the material, but also structural traps due to disorder of the molecules or the polymer conformation (see e.g. [29]). In many cases also the environmental conditions, e.g. oxygen or moisture, have been found to alter the properties of these materials or devices made from them. This requires a great deal of care in preparing the substances and devices and sometimes makes the comparison of results obtained by different groups problematic.

As shown schematically in Fig. 1, electroluminescence in organic solids requires several steps including the injection, transport, capture and radiative recombination of positive and negative charge carriers inside an organic layer with suitable energy gap to yield visible light output. For simplicity we have drawn the spatial variation of the molecular energy levels in a band-like fashion, however, we have to bare in mind again that these organic semiconductors are disordered materials without a well-defined band structure. Also not included in this picture are polaronic effects, i.e. the fact that due to a structural relaxation the energy levels of charged molecules are different from the neutral state levels.

In the absence of doping, interface dipoles and other interfacial effects and assuming vacuum level alignment the energy barrier for charge carrier injection is in first approximation given by the energetic offset between the work functions of the used metals and the energy levels of the organic material. However, care has to be taken when using the work functions of pure metals measured in ultra-high vacuum (UHV) and the energy levels of organic molecules measured by cyclic voltammetry in solution or by photoelectron spectroscopy on nominally pure films in UHV. A first reason is that the preparation conditions of OLEDs are usually not clean enough to exclude the oxidation of low work function metals or the formation of adsorbate layers even on noble metals. A second reason is that chemical reactions between the organic layer and the metals can lead to the formation of an interfacial layer with different properties than the bulk materials which in turn significantly modifies the energetics at the injecting contact [30,31]. Furthermore, the disordered nature of the organic material causes additional peculiarities which have to be taken into account in describing the injection process $[32,33]$.

Once carriers are injected into the organic material these are transported in the applied field towards the counter electrode. Due to the disorder charge carrier transport in organic materials is to be described by hopping between sites with different energy and distance. Additionally, carriers can be intermittently trapped in gap states originating from impurities or structural traps. This results in low carrier mobilities, which are typically between $10^{-3}$ and $10^{-7} \mathrm{~cm}^{2} / \mathrm{Vs}$ at room temperature and in many cases strongly depend on temperature and the magnitude of the applied electric field (see e.g. [34]). With these low carrier mobilities and negligible free carrier densities even in the presence of non-vanishing injection barriers the metal-organic contact may be able to inject more carriers than the organic bulk material has in thermal equilibrium. This will then lead to the formation of space charges, which reduce the electric field at the injecting contact and thus impede further charge carrier injection $[35,36]$.

Finally, for electroluminescence, charge carriers of opposite sign have to recombine and form an exciton, which then decays radiatively. It has been shown that again as a consequence of low carrier mobilities the process of electron-hole capture is diffusion-controlled and is therefore of the Langevin type $[37,38]$. Moreover, the exciton can not only decay radiatively but also radiation-less. Like under optical excitation the ratio of radiative decay depends on the photoluminescence quantum yield, however with the difference that the total spin of the excitons generated by carrier recombination of non-geminate pairs can be a triplet or a singlet state with a branching ratio of $3: 1$, setting an upper limit of $25 \%$ for the internal efficiency of the conversion of injected carriers into 
photons via singlet excitons. It has been demonstrated only recently that by choosing materials with a high yield of short-lived phosphorescence, triplet emission can be harvested for making very efficient organic electroluminescent devices $[39,40]$.

We note that the necessity to use contacts with different work function (usually a high work function metal, like e.g. Au, or a transparent conducting oxide is used as anode and a low work function metal, such as $\mathrm{Ca}$, as cathode) in order to obtain double-carrier injection in OLEDs leads to the presence of a non-negligible built-in voltage $V_{b i}$ $\left(=\Phi_{b i} / q\right.$ in Fig. 1) across the organic layer. (We will use the term built-in voltage although it is also common for the diffusion voltage at semiconductor p-n junctions). Neglecting energy level shifts due to interface dipoles, the built-in voltage is equal to the contact-potential difference of the two metal electrodes. The physical importance of $V_{b i}$ is that it reduces the applied external voltage $V$ such that a net drift current in forward bias direction can only be achieved if $V$ exceeds $V_{b i}$. Thus the knowledge of $V_{b i}$ is crucial and it has to be considered in all equations describing injection and transport in the devices. In the following we will denote $\mathcal{V} \equiv V-V_{b i}$ as the effective voltage across the organic layer under forward bias conditions.

In this paper we will focus on charge carrier injection and transport, because these are the dominant factors to explain the experimentally observed variation of the device current over many orders of magnitude. The recombination step will be discussed subsequently when the results obtained on single and double-carrier devices are compared.

\section{B. Charge carrier injection and transport}

There are two limiting regimes of device operation, namely space-charge limitation and injection limitation of the current. Both have been realized experimentally in OLED structures. The occurrence of space-charge limited currents requires that at least one contact has good injecting properties to provide an inexhaustible carrier reservoir. Injection limitation, by contrast, occurs if the injection barrier is so large that the injection current from the contact into the organic is insufficient to deliver the maximum possible space-charge limited current in the material. At present, even for a given system, there is still an ongoing debate which mechanism prevails: injection limitation at the contacts or transport limitation in the bulk. We will therefore briefly give an overview on some basic concepts for the description of these processes.

Carrier injection into a semiconductor is usually treated either in terms of Fowler-Nordheim (FN) tunneling or Richardson-Schottky (RS) thermionic emission [41]. The latter is based on lowering of the image charge potential by the external field $F=\mathcal{V} / d$. The current density $j_{R S}$ as a function of the field is then given by:

$$
j_{R S}=A^{*} T^{2} \exp \left(-\frac{\Phi_{B}-\beta_{R S} \sqrt{F}}{k_{B} T}\right)
$$

with the Richardson constant $A^{*}=4 \pi q m^{*} k_{B}^{2} / h^{3}$ $\left(=120 \mathrm{~A} /\left(\mathrm{cm}^{2} \mathrm{~K}^{2}\right)\right.$ for $\left.m^{*}=m_{0}\right), \beta_{R S}=$ $\sqrt{q^{3} / 4 \pi \varepsilon \varepsilon_{0}}$ and the zero field injection barrier $\Phi_{B}$. ( $q$ : elementary charge, $m_{0}$ free electron mass, $k_{B}$ : Boltzmann's constant, $h$ : Planck's constant, $\varepsilon$ : relative dielectric constant, $\varepsilon_{0}$ vacuum permittivity). The Fowler-Nordheim mechanism, on the other hand, ignores Coulombic effects and considers mere tunneling through a triangular barrier into continuum states:

$$
\begin{gathered}
j_{F N}=\frac{A^{*} q^{2} F^{2}}{\Phi_{B} \alpha^{2} k_{B}^{2}} \exp \left(-\frac{2 \alpha \Phi_{B}^{3 / 2}}{3 q F}\right) \\
\text { with } \alpha=\frac{4 \pi \sqrt{2 m^{*}}}{h}
\end{gathered}
$$

Both concepts are under certain conditions appropriate in inorganic semiconductors with extended band states and large mean free path, yet one can not expect that they hold in organic semiconductors, where the average mean free path is of the order of the molecular distances. The existence of disorder in organic semiconductors poses an additional obstacle to be overcome by the injected carrier: to move away from the contact into the bulk the carrier has to overcome random energy barriers caused by disorder. This leads to an enhanced backflow of injected carriers into the electrode. This process of injection into a disordered hopping system has been studied analytically [42] and by Monte Carlo simulations [32,33]. The simulations by Wolf et al. show that, although this injection mechanism resembles RS thermionic emission, quantitative differences exist concerning the field and temperature dependence as well as the absolute value of the current, which is found by orders of magnitude lower than predicted by the Richardson constant [33].

Space-charge limited currents (SCLC) in a device can occur if at least one contact is able to inject locally higher carrier densities than the material has in thermal equilibrium without carrier injection. The problem of SCLC in insulators has been extensively treated by Lampert and Mark [35]. Therefore we will list only briefly the basic equations that govern the steady-state behaviour of unipolar space-charge limited current flow (here given for electrons). These are the drift-diffusion equation (3), the Poisson equation (4), the continuity equation (5) together with the boundary condition (6) and the equations relating free and trapped charge carrier densities $(7,8)$. Due to the device geometry a one-dimensional treatment is sufficient. Furthermore, we will use the convention $\vec{j}=-j \vec{e}_{x}$ and $\vec{F}=-F \vec{e}_{x}$ where $\vec{e}_{x}$ is a unit vector in $x$-direction and the injecting contact is at the position $x=0$. 


$$
\begin{aligned}
j & =q n \mu F-q D \frac{\mathrm{d} n}{\mathrm{~d} x} \\
\frac{\varepsilon \varepsilon_{0}}{q} \frac{\mathrm{d} F}{\mathrm{~d} x} & =\left(n-n_{0}\right)+\sum_{j}\left(n_{t_{j}}-n_{t_{j}, 0}\right) \\
\frac{\mathrm{d} j}{\mathrm{~d} x} & =0 \\
\mathcal{V} & \equiv V-V_{b i}=\int_{0}^{d} F \mathrm{~d} x \\
n & =N_{C} \exp \frac{E_{F}-E_{C}}{k_{B} T} \\
n_{t_{j}} & =N_{t_{j}}\left[1+g_{j}^{-1} \exp \frac{E_{t_{j}}-E_{F}}{k_{B} T}\right]^{-1}
\end{aligned}
$$

$\mu$ is the carrier mobility, $D$ the diffusion coefficient, $d$ the device thickness, $N_{C}$ the density of states in the conduction band, $E_{C}$ the conduction band energy and $E_{F}$ the position of the quasi-Fermi level. $n$ and $n_{t, j}$ denote the spatially varying free and trapped electron concentration in the $j$ th set of traps with energy $E_{t_{j}}$ and degeneracy $g_{j}$ (in most cases $g_{j}=1$ ), respectively, and $n_{0}$ and $n_{t_{j}, 0}$ are the corresponding thermal equilibrium values. If traps are distributed in energy rather than in discrete levels then the sum has to be replaced by an integral over the energy. A frequently used distribution in the context of organic semiconductors is an exponential one with a total trap density $N_{t}$ and a characteristic decay energy $E_{t}$ :

$$
H_{t}(E)=\frac{N_{t}}{E_{t}} \exp \frac{E-E_{C}}{E_{t}} \quad\left(E \leq E_{C}\right)
$$

Then instead of Eq. (8) one has:

$$
n_{t}=\int_{-\infty}^{E_{C}} \frac{H_{t}(E)}{1+g^{-1} \exp \left[\left(E-E_{F}\right) / k_{B} T\right]} \mathrm{d} E
$$

This set of differential equations has analytical solutions only for some special cases. In the case of a perfect insulator without intrinsic carriers and traps and for a charge carrier mobility independent of the electric field, the SCL current obeys the Mott-Gurney equation [43]:

$$
j_{S C L C}=\frac{9}{8} \varepsilon \varepsilon_{0} \mu \frac{\mathcal{V}^{2}}{d^{3}}
$$

This equation is derived neglecting diffusion and with the boundary condition that the electric field at the injecting contact vanishes, which leads to an infinitely high carrier density at the contact. The inclusion of the diffusion term resolves the problem of the infinite carrier concentration, but makes an analytic solution of the system of differential equations impossible (see [5] for a discussion of numerical solutions). However, the treatment of diffusion is not straightforward since the validity of the Einstein relation between the diffusion coefficient and the mobility has not been established yet in disordered organic solids [44]. The importance of the trap-free SCL current is that it is the maximum possible unipolar current a sample can sustain at a given applied potential difference. A current in excess of this value is only possible in the case of double-carrier injection where charges of opposite sign are mutually able to compensate part of the space-charge.

In the presence of traps the current is in general lower and the quadratic field dependence is retained in the case of discrete trap levels only (or when all traps are filled). Then Eq. (11) has to be modified by a factor $\theta=n /\left(n+n_{t}\right)$ equal to the ratio of free carriers to the total number of carriers. If traps are distributed in energy they will be gradually filled with increasing electric field (i.e. $\theta$ depends on the electric field) and the current will increase faster than quadratic until all traps are filled. The problem has been solved analytically for the above given exponential trap distribution $H_{t}(E)$. In this case the so-called trapcharge limited current (TCLC) with the parameter $l=E_{t} / k_{B} T$ derived from the trap distribution is given by $[35,36]$ :

$$
j_{T C L C}=N_{C} \mu q\left(\frac{\varepsilon \varepsilon_{0} l}{N_{t} q(l+1)}\right)^{l}\left(\frac{2 l+1}{l+1}\right)^{l+1} \frac{\mathcal{V}^{l+1}}{d^{2 l+1}}
$$

If the assumption of a field-independent charge carrier mobility is dropped, an analytic solution for arbitrary $\mu(F)$ dependence is still possible in the absence of traps, however, $j(\mathcal{V})$ can then be given in parametric form only [45]. An approximate analytical solution has been derived by Murgatroyd [46] for the so-called Poole-Frenkel field dependence of the mobility:

$$
\mu(F)=\mu_{0} \exp (\beta \sqrt{F})
$$

which is very frequently observed in amorphous molecular materials, molecularly doped polymers and also most conjugated polymers [34]. The current density in this case is approximately the trapfree SCL current multiplied with the Poole-Frenkel mobility [46]:

$$
j_{S C L C}^{(P F)} \approx \frac{9}{8} \varepsilon \varepsilon_{0} \mu_{0} \frac{\mathcal{V}^{2}}{d^{3}} \exp (0.89 \beta \sqrt{\mathcal{V} / d})
$$

Another analytic expression has been given for a mobility following a power law $\mu(F)=\mu_{0}\left(F / F_{0}\right)^{n}$ [47]:

$$
j_{S C L C}^{(P L)}=\varepsilon \varepsilon_{0} \mu_{0} \frac{[1 /(n+3)]^{n+2}}{F_{0}^{n}(n+2)} \frac{\mathcal{V}^{n+2}}{d^{n+3}}
$$

which also yields a power law behaviour of the current like the TCLC expression, however, the thickness dependence at a given exponent ( $n$ respectively $l$ ) is different. If both the presence of traps and a field-dependent mobility are included, in general, only numerical solutions of the problem are possible. 
The situation becomes even more complicated in real OLEDs where double-carrier injection and recombination occurs. It has been demonstrated that recombination in organic LEDs is a bimolecular process following the Langevin theory $[6,37,38,48,49]$, because it is based on a diffusive motion of positive and negative carriers in the attractive mutual Coulomb field. This implies that the recombination constant $R$ is proportional to the carrier mobility:

$$
R=\frac{q}{\varepsilon \varepsilon_{0}}\left(\mu_{h}+\mu_{e}\right)
$$

The inclusion of double-carrier injection and recombination couples the respective sets of transport equations for electrons and holes, since the Poisson equation now contains both the densities of electrons $(n)$ and holes $(p)$ and the continuity equation becomes

$$
\frac{\mathrm{d} j_{e}}{\mathrm{~d} x}=-\frac{\mathrm{d} j_{h}}{\mathrm{~d} x}=q R n p
$$

Therefore analytical solutions for the electron, hole and recombination currents as a function of voltage are only possible under simplifying assumptions like a mobility independent of the electric field and the absence of diffusion and trapping. E.g. Scott et al. have extended the Parmenter-Ruppel solution [35] by including Langevin recombination [50]. Numerical solutions for different cases have also been obtained on OLEDs by various authors [48,51-53], however due to the complexity of the numeric procedures the application to the analysis of experimental data is not straightforward.

In the following we will analyze our experimental results which were mainly obtained on singlecarrier devices using approximate analytic equations or simple numeric integration of the abovementioned transport equations, neglecting diffusion, but including a field-dependent carrier mobility and distributed trap states. A central question thereby will be whether the current in a device is injection or space-charge limited, and, if the latter case applies, whether TCLC with an exponential trap distribution or a field and temperature dependent charge carrier mobility play the dominant role. We will show that apart from the dependence of the current on voltage and temperature, which have already been investigated in these devices, the dependence on the thickness of the organic layer provides a unique criterion to distinguish between the underlying mechanism. For clarity we therefore briefly list the functional dependence of the current on the thickness at constant electric field for these three situations:

(i) for purely injection limited behaviour (regardless what the actual mechanism is in detail) the current at constant field has no explicit thickness dependence (Eqs. 1,2):

$$
j=j(F)
$$

(ii) for trap-free space-charge limited conduction with (or without) a field-dependent mobility the current at constant field scales with $d^{-1}$ (Eqs. 11, 14, 15):

$$
j=\tilde{j}(F) / d
$$

(iii) for trap-charge limited conduction with an exponential trap distribution and a fieldindependent mobility the current at constant field scales with $d^{-l}$ with $l>1$ (Eq. 12):

$$
j=\tilde{\tilde{j}}(F) / d^{l}
$$

In order to prove that Eq. (19) also holds for a strongly field-dependent carrier mobility, where the Murgatroyd approximation becomes less accurate, we have shown in Fig. 2 numerically simulated $j-V$ characteristics for trap-free SCLC with a mobility $\mu(F)=\mu_{0} \exp (\beta \sqrt{F})$ for two different parameter sets of $\mu_{0}$ and $\beta$. It is clearly seen that the dependence $j(F=$ const. $) \propto d^{-1}$ is fulfilled even for large values of $\beta$ where the current is far from being quadratic in the voltage.

\section{MATERIALS, DEVICE FABRICATION AND EXPERIMENTAL METHODS}

As already mentioned in the introduction, the characteristic feature of OLEDs based on molecular materials is the usage of a hetero junction between a hole conducting and an electron conducting material sandwiched between an anode and a cathode contact for hole and electron injection, respectively. Fig. 3 shows the typical device structure used here for the investigation of the electrical characteristics of devices with one or more organic layers. Our studies were focused on the common combination of aromatic amines like TPD or NPB as hole transporters and Alq as electron transport and light-emitting material (see Fig. 4). The materials were commercially available: TPD and NPB from Syntec, Alq from Dojindo and Aldrich. Different types of Alq have been used: Alq(1) was multiply sublimed, Alq(2) (98\% Alq content) and Alq(3) (99.995\% Alq content) were used as obtained. Fig. 5 shows a schematic energy level diagram of such a hetero-layer device with indium-tin oxide (ITO) as transparent hole injecting and $\mathrm{Ca}$ as electron injecting contact. However, we note that these energy values have to be taken with care since they are usually obtained on the isolated compounds under conditions different from those used for the preparation of OLEDs. Furthermore, energetic details at the interfaces which depend on the amount of inter-diffusion, on possible chemical reactions and on the extent of interface dipole formation are not included in this drawing (see e.g. [30]). The 
charge carrier mobility of TPD (or similarly NPB) and of Alq as determined by time-of-flight measurements is shown in Fig. 6. The hole transport in TPD is well characterized [34] and displays relatively high mobility values with only a weak field dependence. For Alq the electron mobility is much lower and exhibits a strong dependence on the electric field $[54,55]$ typical for dispersive hopping transport. As we will describe later, this strong field dependence of the mobility can be a source of ambiguity in the interpretation of the electrical device behaviour.

For our investigations we used two types of substrates. One type were ITO coated glass substrates from Merck $(20-80 \Omega / \square)$, where the ITO was patterned by a photoresist technique to define anode contacts. These substrates were used for hole-only and bipolar devices. The other type were glass substrates with evaporated stripes of $\mathrm{Al}$ for electrononly devices. The ITO substrates were thoroughly cleaned in an ultrasonic bath with different solvents and thereafter exposed to an oxygen plasma to remove residual solvents and to enhance the work function of the ITO.

The importance of the plasma treatment on the device performance can be seen from Fig. 7, where current and luminance of otherwise identically treated devices are compared. The plasma treatment not only reduces the drive voltage considerably, it also suppresses the occurrence of current anomalies at low voltage [56]. Although the mechanism behind these effects is not yet understood in detail, it is meanwhile generally accepted that some kind of oxidative treatment of the ITO or other high work function anode materials is required to improve hole injection and to arrive at reproducible device characteristics [57-60]. Immediately after plasma treatment the substrates were transferred via a nitrogen-filled glovebox system into the evaporation chamber, which can be equipped with up to 10 thermal evaporation sources for the deposition of organic materials and metals with low evaporation temperature. Depositions were carried out at a base pressure of about $10^{-6} \mathrm{mbar}$ with rates of $1 \AA / \mathrm{s}$. Via a rotating substrate holder with exchangable shadow masks several devices with controlled variation of the layer thickness or layer sequence could be fabricated in the same vacuum process. The metal cathodes $(\mathrm{Ca}$ or $\mathrm{Al})$ were deposited on top of the organic layers without breaking the vacuum. $\mathrm{Ca}$ cathodes were covered with a Mg:Al:Zn-alloy to protect them from degradation under air during short-term transfer between different measurement systems. From the evaporator the samples were then taken back to the glovebox system where they were characterized and stored under inert atmosphere (less than 1ppm moisture and oxygen). No detectable changes of the device characteristics were observed after several months of storage under these conditions.
Current-voltage $(I-V)$ and luminance-voltage $(L-V)$ characteristics were measured inside the glovebox with a Hewlett Packard semiconductor parameter analyzer (HP 4155B) and a Si photodiode (Hamamatsu S2281) in front of the lightemitting pixel. The OLED was thereby kept in a modified HP 16442A test fixture. With this setup currents as low as $10^{-13} \mathrm{~A}$ can be reliably measured. The luminance calibration of the photodiode was obtained with a Minolta CS-100 luminance meter. Additionally we measured the total amount of emitted light from OLEDs by using an integrating sphere with a calibrated Si photodiode coupled to a power meter (Gigahertz). From the photodiode current together with the spectral sensitivity of the photodiode and with the EL spectrum of the OLED we obtained the total emitted light intensity and from this quantity the EL quantum efficiency $\eta_{E L}$. We note that this determination of the external quantum efficiency yields about a factor of 2 higher values than the ones obtained by using the forward emitted luminance values (for the calculation of $\eta_{E L}$ from $L$ see Ref. [61]). The photoluminescence quantum efficiency was determined on films deposited on fused silica following a procedure suggested by de Mello et al. [62]. Thereby the absorption and emission spectra were simultaneously measured in an integrating sphere under excitation with a GaN LED (peak wavelength of $370 \mathrm{~nm}$ ) using a spectrograph coupled to a CCD array. Transient electroluminescence was measured using a pulse generator (Berkeley Nucleonics) as source of rectangular voltage pulses and a photomultiplier (Hamamatsu 5783-01) for detection of light-emission. Voltage, current and EL traces were monitored by a digitizing oscilloscope (Tektronix TDS754C). To avoid an influence of trapped charges from the preceding pulse on the transient response we used single voltage pulses and sufficient waiting time between subsequent pulses. Additionally, in measurements at low temperature samples were short-circuited and warmed up to room temperature after each pulse to accelerate discharging. Impedance measurements were carried out with an impedance analyzer (Hewlett Packard HP 4194A, $100 \mathrm{~Hz}-15 \mathrm{MHz}$ ) or a frequency response analyzer (Solartron Instruments SI $1260 \mathrm{~A}, 10 \mu \mathrm{Hz}-10 \mathrm{MHz}$ ) using an oscillator amplitude of no more than $100 \mathrm{mV}$. For temperature dependent characterization a liquid-nitrogen cooled optical cryostat (Cryovac) with static heat exchange gas was used in combination with the setup described above. 


\section{EXPERIMENTAL RESULTS AND DISCUSSION}

\section{A. Injection vs. bulk limited conduction in organic light-emitting devices}

\section{Hole transporting single-layer devices}

The hole transport properties of aromatic diamines like TPD or NPB have been investigated intensively in the past due to their application in xerography (for a review see [34]). In this type of application excess charge carriers are generated by absorption of light in a charge carrier generation layer, a few microns thick, and a fast transport of the positive carriers through an insulating layer to a collecting electrode is required. For high-speed copying, the most important material parameter is the charge carrier mobility. This property has been well characterized by transient photocurrents (time-of-flight technique). Also dark injection currents have been studied in some diamines and consistent mobility values have been achieved from d.c. or transient space-charge limited currents if sufficiently well injecting contacts, like carbon-filled polymers, were used [47].

In OLEDs, however, the typical organic layer thickness is much less (100nm or below), which leads to much more stringent requirements for low injection barriers at the injecting electrodes in order to prevent that the current is injection limited. Given the need to use a transparent contact for coupling out light in OLEDs, a very frequent choice for the hole injecting anode material is indium-tin oxide. It has been reported already by Giebeler et al. and Campbell et al. that single-layer devices of TPD on ITO suffer from injection limitation $[63,64]$. We will demonstrate here that orders of magnitude higher current densities can be achieved when oxygen-plasma treated ITO is used, but that, nevertheless, the question of bulk or injection limitation deserves attention. Before addressing this issue, however, we will discuss some general features of the $I-V$ characteristics.

Fig. 8 shows $I-V$ characteristics of an ITO/NPB/Ca device (NPB thickness of about $430 \mathrm{~nm}$ ) measured in steps of $0.1 \mathrm{~V}$ between $-7 \mathrm{~V}$ and $+7 \mathrm{~V}$ and vice versa with different delay times. Below the turn-on voltage at about $2 \mathrm{~V}$ a large hysteresis of the current is visible between both sweep directions for short delay times. Especially the voltage where the current passes through zero is drastically affected by the sweep direction. This effect can be gradually reduced if a longer delay time between the voltage steps is chosen. With increasing delay time the current in reverse direction decreases and the hysteresis becomes smaller. However, to achieve almost identical curves in both sweep directions an extremely long delay time of 300 s is required. We will show in a forthcoming publication [65] that the transient behaviour of the
$I-V$ characteristics can be simulated by taking into account leakage currents together with deep traps which require large time constants for charging and discharging in response to a change of the external bias. This is in qualitative agreement with transient studies of deep traps in polymer LEDs by Campbell and Bradley [66]. An important consequence of this hysteresis is that useful information from the reverse bias behaviour of the devices can only be obtained if the measurement speed is extremely slow.

On the other hand the current in forward direction is virtually not affected by the different measurement speed. The small increase at high voltages with increasing delay time may be ascribed to slight heating of the device which has a noticeable effect due to the strong temperature dependence of the $I-V$ characteristics. If the $I-V$ characteristics measured with 300 s delay time (Fig. 9) is taken as an equilibrium curve then one can derive further properties of these devices. First the rectification ratio between forward and reverse bias is more than 7 orders of magnitude at $|V|=6 \mathrm{~V}$ and increases further at higher voltage. This is among the highest values reported in the literature [67]. The current in reverse direction is very low with only a few pA. We note that it is not trivial to achieve such low currents in devices with thin organic layers $(d<100 \mathrm{~nm})$ in view of the ITO surface roughness which can amount up to a few ten nanometers [68] and because of imperfections of the substrate cleaning process. As shown in the inset of Fig. 9 the current in reverse direction varies almost linearly with the applied voltage. Although it cannot be ruled out that the ohmic behaviour of the current may be due to leakage, it can be used to estimate an upper limit of the intrinsic carrier concentration. Assuming ohmic conduction, from the obtained value of the conductivity $\sigma=q n_{0} \mu \approx 4.4 \times 10^{-16}(\Omega \mathrm{cm})^{-1}$ in this bias range and with a mobility $\mu$ of about $10^{-4} \mathrm{~cm}^{2} / \mathrm{Vs}$ an upper limit of the intrinsic carrier concentration $n_{0}<3 \times 10^{7} \mathrm{~cm}^{-3}$ can be calculated. Given the large energy gap of about $3 \mathrm{eV}$ such a low value of $n_{0}$ is understandable. Thus these devices are fully depleted and will not show band bending effects (apart from possible interfacial dipoles on a molecular length scale).

Looking at the forward bias behaviour one first notices that the steep increase of the current does not begin at $0 \mathrm{~V}$ but at a turn-on voltage of about $2 \mathrm{~V}$. As already discussed in section II this voltage can be identified with the built-in voltage $V_{b i}$ necessary to overcome the contact potential difference between the two electrodes. With respect to the built-in voltage the device is effectively forward biased when the applied voltage exceeds $V_{b i}$ and reverse biased for $V<V_{b i}$. We found that within an uncertainty of 0.1 to $0.2 \mathrm{eV}$ the built-in voltage is independent of the organic material (Alq single-layer and also NPB/Alq hetero-layer devices 
with ITO and Ca electrodes yield the same value) and the thickness of the respective organic layer. It is therefore a quantity depending mainly on the work function difference of the electrode materials. Taking the work function of $\mathrm{Ca}$ as $3 \mathrm{eV}$ one can estimate a value of about $5 \mathrm{eV}$ for ITO. This indicates that the oxygen plasma treatment of ITO enhances its work function significantly $[69,70]$. We have also verified the agreement of the values of $V_{b i}$ obtained from $I-V$ characteristics with the open circuit photovoltage under white-light illumination, which has been suggested as a measure of the built-in voltage [71].

Above $V_{b i}$ the current increases quasiexponentially over many orders of magnitude in a narrow voltage range between 2 and $3 \mathrm{~V}$ and then continues growing with reduced slope. As mentioned above, the important question in the context of OLEDs is whether the current above $V_{b i}$ is injection or space-charge limited. The latter means that the current should approximately follow the Mott-Gurney equation (11) for trap-free space charge limited currents since NPB is known to display trap-free hole transport with only very weak dependence of the mobility on the electric field [72]. However, in previous work on OLEDs it was found that NPB and TPD on ITO display purely injection limited behaviour [63,64]. In a device with $100 \mathrm{~nm}$ NPB sandwiched between ITO and Ag electrodes current densities of only $10^{-5} \mathrm{~A} / \mathrm{cm}^{2}$ were reported at $V=6 \mathrm{~V}$ [63], which is more than 2 orders of magnitude lower than the value obtained here for a much thicker organic layer. Yet, this is not enough to conclude that the behaviour in our devices is space-charge limited. Another criterion is the thickness dependence of the current, which is shown in Fig. 10. Here we have chosen $\mathrm{Al}$ as cathode, therefore a built-in voltage of about $0.9 \mathrm{~V}$ was considered in the analysis. Already from the raw data (Fig. 10(a)) it can be seen that the thickness dependence of the current is comparatively weak, which indicates that a dependence of $j \propto \mathcal{V}^{2} / d^{3}$ required for SCLC may probably not apply. This becomes more clear from the double-logarithmic representation in Fig. 10(b), where SCLC should lead to straight lines with a slope of 2 . If a field-dependent mobility of the form $\mu=\mu_{0} \exp (\beta \sqrt{F})$ is taken into account the $j-V$ dependence of the thickest sample can be simulated numerically above $\mathcal{V} \approx 2 \mathrm{~V}$ with trapfree SCLC and values of $\mu_{0}=10^{-4} \mathrm{~cm}^{2} / \mathrm{Vs}$ and $\beta=0.0015(\mathrm{~cm} / \mathrm{V})^{1 / 2}$. Published mobility data of NPB have the same value of $\beta$ however $\mu_{0}$ is a factor of 3 to 6 larger [53,73]. Such a difference in the obtained $\mu_{0}$ values could tentatively be explained by slight differences of the film growth conditions, which are known to have an influence on the mobility [74]. However, using the same parameters to simulate the $j-V$ dependence of the device with a $68 \mathrm{~nm}$ thick NPB layer leads to a difference to the experimental data of more than a factor of
10. Therefore, another possibility has to be taken into account, namely that the current is injection limited. For pure contact limitation, however, regardless what the injection mechanism actually is, it should be possible to express the current as a function of the electric field alone, without an explicit thickness dependence. Thus when plotted versus the effective field $F=\mathcal{V} / d$ all curves should be identical. As shown in Fig. 10(c) this is not the case, there is about a factor of 4 difference between the thickest and the thinnest sample.

These results suggest that NPB single-layer devices on ITO are neither purely injection nor purely space-charge limited. Depending on the organic layer thickness contact limitation for thin layers or bulk limitation for thick layers seem to play the more dominant role. This is consistent with previous work in the literature, where trapfree space-charge limited currents could be obtained in films with thickness of about $10 \mu \mathrm{m}$ on a carbon-filled polymer substrate [47]. With ITO substrates SCLC has only been reported for several hundred nanometers thick layers of starburst amines (m-MTDATA, naph-DATA) [63,75], which on the other hand have considerably lower hole mobility $\left(\mu_{0} \approx 10^{-5} \mathrm{~cm}^{2} / \mathrm{Vs}, \beta \approx 0.003(\mathrm{~cm} / \mathrm{V})^{1 / 2}\right)$ and lower ionization potential, both factors facilitating the achievement of SCLC in these devices. Recently, Wolf et al. suggested a criterion to discriminate between injection and space-charge limitation based on Monte Carlo simulations of the injection yield from a metal into a disordered molecular solid as a function of the injection barrier [76]. They find that at an electric field of $10^{6} \mathrm{~V} / \mathrm{cm}$ with a charge carrier mobility of $10^{-4} \mathrm{~cm}^{2} / \mathrm{Vs}$ the allowable injection barrier to achieve SCLC, i.e. zero electric field at the injecting contact, is only about $0.12 \mathrm{eV}$. If the barrier is $0.1 \mathrm{eV}$ higher the field at the injecting contact is already $10^{5} \mathrm{~V} / \mathrm{cm}$ which results in so-called space-charge perturbed injection limited currents. Given the ionization potential of about $5.4 \mathrm{eV}$ of NPB this would require a work function of ITO significantly higher than $5 \mathrm{eV}$, which has never been obtained, not even after different oxidative treatments $[57,58,68]$.

Thus one has to conclude that in NPB singlelayer devices with thin NPB layers $(d<100 \mathrm{~nm})$ charge carrier injection is the current limiting factor. However, since in hetero-layer devices with Alq holes are blocked at the organic-organic interface and since the electron mobility in Alq is much lower than the hole mobility in NPB, the injection limited current from ITO into NPB may still be sufficient to fulfill the demand of holes in these devices.

\section{Electron transporting single-layer devices}

Although Alq is one of the most widely used materials in OLEDs based on small molecules it is still a relatively ill characterized material con- 
cerning electronic and optical properties as compared to the huge amount of work on polymers or molecular hole transporters (for reviews see e.g. $[26,34,77])$. Only recently, topics such as quantum chemical calculations, crystal structure, and the influence of film growth conditions and purity on the electrical properties have been addressed in the literature [74,78,79]. Moreover, different groups world-wide are working with material from different sources (commercial or academic) and it is not yet clear that all of these materials show the same properties especially concerning the electrical properties. Therefore we have compared $I-V$ characteristics of different commercially available Alq material. Fig. 11 shows data of electron-only devices $(\mathrm{Al} / \mathrm{Alq} / \mathrm{Ca}$ ) with Alq obtained from Dojindo $(\mathrm{Alq}(1))$ and two different types from Aldrich $(\operatorname{Alq}(2)$ and $A l q(3))$. Besides, we have also tested other commercially available Alq and material synthesized or purified by other laboratories. They were all found to be between the two extremes displayed in Fig. 11. The devices were built on Al anodes to avoid double-carrier injection, which was verified by the absence of a detectable electroluminescence signal. Clearly, between the materials huge differences of the current with variations of more than 3 orders of magnitude at $8 \mathrm{~V}$ can be observed. The highest current density was achieved with $\mathrm{Alq}(1)$. The values at $8 \mathrm{~V}$, equivalent to an electric field of $0.8 \mathrm{MV} / \mathrm{cm}$ (taking into account a built-in voltage of $0.7 \mathrm{~V}$ ), are comparable to published results [80]. Differences also exist in the onset voltage which is only $0.7 \mathrm{~V}$ for $\mathrm{Alq}(1)$ and increases above $1 \mathrm{~V}$ for $\mathrm{Alq}(2)$ and $\mathrm{Alq}(3)$. Additionally, the tendency to develop leakage currents was found to be closely correlated to the behaviour in forward direction. While $\mathrm{Alq}(1)$ shows extremely low reverse current comparable to the NPB devices discussed before, the other Alqs have considerably higher leakage currents below $-1 \mathrm{~V}$. For $\mathrm{Alq}(3)$ this is even true for the forward direction since the bump in the current between 1 and $6 \mathrm{~V}$ has to be ascribed to leakage currents. The doublelogarithmic plot in Fig. 11(b) yields a power law behaviour of the current $j \propto \mathcal{V}^{l+1}$ with different exponents $(l+1)$. Such power laws with exponents larger than 2 have been interpreted as indication for trap-charge limited conduction [81]. Based on this interpretation the increase of $(l+1)$ would then indicate that the trap depth and density increase from $\mathrm{Alq}(1)$ to $\mathrm{Alq}(3)$. However, as long as no quantitative information about the content and nature of impurities and traps in the materials and the detailed morphology of the films and its dependence on the film growth conditions is available, it is not possible to give the reasons for the largely different behaviour. Therefore in the following we will mainly discuss devices with $\operatorname{Alq}(1)$ where the highest currents were achieved.

The current-voltage characteristics of Alq devices have been investigated by several groups be- fore, however, their interpretations have been controversial, ranging from purely injection limited to purely bulk limited conduction. Burrows et al. have reported the first extended studies of temperature and thickness dependent $I-V$ characteristics in ITO/TPD/Alq/Mg: $\mathrm{Ag}$ devices [81]. From the experimentally observed power law dependence of the current $j \propto V^{l+1}$ with $l>1$ they concluded that the device characteristics was determined by the Alq layer and that the dominant mechanism was trap-charge limited conduction with an exponential distribution of traps (with a characteristic energy $E_{t}=0.15 \mathrm{eV}$ ). Based on these findings Shen et al. performed numerical studies of trap-charge limited currents (TCLC) in single and double-carrier devices [52]. Stößel et al. also reported TCLC in their $\mathrm{Mg} / \mathrm{Alq} / \mathrm{LiF} / \mathrm{Al}$ devices with $E_{t}=0.11 \mathrm{eV}$ [82]. Previously, we have also used this model and derived a trap energy of $200 \mathrm{meV}$ for devices with $\mathrm{Alq}(2)$. It was noticed, however, that the observed thickness dependence of the current did not agree with the predictions of TCLC [83]. The application of the TCLC model to these materials can be criticized since the analysis requires a constant charge carrier mobility, which is not fulfilled here. Like many disordered molecular solids and polymers, Alq displays a pronounced field and temperature dependence of the mobility (see also section IVB and $[54,55,84])$. As pointed out by Ioannidis et al. [85] the obtained values of the trap energy in the range of 110 to $150 \mathrm{meV}$ could equally well be assigned to a distribution of transport states which is usually described as a Gaussian with a typical width of $100 \mathrm{meV}$. Thus, these authors described the room temperature $I-V$ characteristics of their $\mathrm{Al} / \mathrm{Alq} / \mathrm{LiF} / \mathrm{Al}$ devices simply by using trap-free SCLC with a field-dependent charge carrier mobility [85]. On the other hand, Barth et al. found that the $I-V$ characteristics of their Al/Alq/Mg:Ag devices were injection limited with an injection barrier of $0.5 \mathrm{eV}$ [80]. The underlying concept of charge carrier injection from a metal into a disordered hopping system has been developed by Gartstein and Conwell [32] and by Bässler's group [33,86], who calculated the injection yield on the basis of Monte-Carlo simulations. Although it was not possible to calculate the absolute value of the injection current by this procedure, the field dependence was predicted to resemble Richardson-Schottky (RS) thermionic emission, however, with a modified RS coefficient and temperature dependence. Injection limitation was also reported by Campbell et al. on $\mathrm{Ca} / \mathrm{Alq} / \mathrm{Ca}$ devices with a barrier of $0.6 \mathrm{eV}$ [87].

This brief and by far not complete overview of experimental investigations and description of electrical transport in Alq devices given here, shows that it is obviously not unambiguous to identify the dominant current limiting mechanism (see also Ref. [88] for a discussion of this issue in polymer LEDs). Such an assignment becomes in general 
more difficult if the range of experimental data is too limited or if important parameters like the thickness dependence $[80,82]$ or the temperature dependence of the current [85,87], the field and temperature dependent mobility [52,81-83], the built-in voltage $[81,85]$ or the influence of doublecarrier injection in a bipolar device $[81,83]$ are not taken into account.

From the above said it is obvious that experiments have to cover a large range of organic layer thickness and temperature and probably different electrode materials in order to be able to distinguish between different models. In the following we will show experimental data for $\mathrm{Al} / \mathrm{Alq} / \mathrm{Ca}$ single-layer electron-only devices as a function of organic layer thickness and temperature and will discuss these data in the framework of the above mentioned competing models. We will demonstrate that especially the thickness dependence provides a unique criterion to decide which mechanism prevails. We will also present simulations of the $I-V$ characteristics based on numerical solutions of the transport equations in section II.

The thickness dependence of the $I-V$ characteristics has been investigated in a series of nine devices made from $\mathrm{Alq}(1)$ with thicknesses ranging from about 100 to $350 \mathrm{~nm}$, all fabricated in one evaporation run. Fig. 12 shows the $I-V$ characteristics of three selected devices in different representations. As compared to our former investigations on $\operatorname{ITO} / \mathrm{Alq}(2) / \mathrm{Ca}$ devices [83] the currents with $\mathrm{Alq}(1)$ are considerably higher and the thickness dependence is much weaker. For purely injection limited behaviour the current plotted versus the electric field should be identical for different organic layer thicknesses and should, according to the simulations by Wolf et al., follow a RS-like linear dependence of $\log (j)$ on the square root of the electric field. As is clearly seen in Fig. 12(a) the current does not obey such a linear dependence on $\sqrt{F}$ and the curves are not identical but differ by a factor of 3 between 100 and 300nm. From the double-logarithmic plot (Fig. 12(b)) one can see that the current approximately obeys a power law $j \propto \mathcal{V}^{l+1}$ with $(l+1)$ between 3.4 and 4.4 for different thickness. This behaviour could be taken as an indication for trap-charge limited conduction (cf. Eq. 12).

However, as already pointed out by Wolf et al. [33], the voltage dependence of the diode current alone is insufficient to distinguish between different mechanisms. As outlined in section II, the thickness dependence of the current at constant electric field provides a much clearer criterion to make such a distinction. In Fig. 13 we have therefore plotted the current at two different values of the electric field versus the Alq thickness. If the current were purely injection limited, it could be expressed as a function of the electric field alone without an explicit thickness dependence (Eq. 18). On the other hand, if it were trap-charge limited with an exponential trap distribution, it should obey a power law of the form $j \propto \mathcal{V}^{l+1} / d^{2 l+1}=F^{l+1} / d^{l}$ (Eq. 20). Finally, if trap-free SCLC (with a fielddependent mobility) prevails, the current should be proportional to $d^{-1}$ (Eq. 19). The high-field data (at $F=0.5 \mathrm{MV} / \mathrm{cm}$ ) in Fig. 13 show that the latter case exactly holds for 6 devices with thicknesses ranging from 350 to about $200 \mathrm{~nm}$. The data points for the 3 thinnest devices (with thicknesses around 100nm) are below the fitted curve, which can be taken as an indication for an influence of the injecting contact at these thicknesses. The inset of Fig. 13 shows the current at lower electric field $(F=0.1 \mathrm{MV} / \mathrm{cm})$ plotted vs. $d^{-3}$, which is the behaviour expected for TCLC with $(l+1)=4$ (cf. Fig. 12(b)). Again a linear dependence is observed in this representation for device thicknesses larger than about 200nm. This observation implies that at low fields charge carrier traps are involved, which become filled at higher electric fields. However, since the $I-V$ curves do not show any step-like structures (expected for filling of traps at discrete energy levels) these traps have to be distributed in energy.

The necessity to include traps is also seen in Fig. 14 where we have compared experimental data of the current for $d \approx 100,200$ and $300 \mathrm{~nm}$ with two different numerical simulations. One is based on trap-free SCLC including a PooleFrenkel type mobility $\mu(F)=\mu_{0} \exp (\beta \sqrt{F})$ with $\mu_{0}=2 \times 10^{-9} \mathrm{~cm}^{2} / \mathrm{Vs}$ and $\beta=0.008(\mathrm{~cm} / \mathrm{V})^{1 / 2}$ (dashed lines). The second one uses the same mobility parameters, but in addition an exponential trap distribution (Eq. (9)) with $E_{t}=0.14 \mathrm{eV}$ and $N_{t}=1.3 \times 10^{17} \mathrm{~cm}^{-3}$ is included (solid lines). For the thinnest device there are only small differences between the two simulations and both agree to within a factor of 2 with the experimental data. Again, the lower experimental values indicate an influence of the injection current at a thickness of $100 \mathrm{~nm}$, but still SCLC is the dominant mechanism. For a thickness of $200 \mathrm{~nm}$ there are noticeable deviations of the trap-free simulation to the experimental data at low voltages, which become more pronounced for the 300nm thick device. However, when an exponential trap distribution is included in the simulations, the data can be perfectly simulated even for the thick devices using the above given parameters. This thickness dependence of the $I-V$ characteristics can be explained by trap filling. It is known that for discrete trap levels with a given trap density the crossover voltage $V_{T F L}$ between the trap-dominated regime at low voltage and the trap-filled SCL regime at high voltage scales with the square of the device thickness [35]: $V_{T F L} \propto d^{2}$. Although no sharp trap-filling voltage is observed for traps distributed in energy, the behaviour of trap-filling with the device thickness is qualitatively the same. Thus one can expect to see an increasing influence of traps for thicker organic films. On the other hand, reducing the thick- 
ness by a factor of 3 (from 300 to 100nm) will decrease $V_{T F L}$ by almost an order of magnitude, which means that for a device with $100 \mathrm{~nm}$ thickness virtually all traps could be filled already at a relatively small voltage above the built-in voltage.

Thus from the thickness dependence of the current at room temperature one can come to the conclusion that the electron current in Alq devices is predominantly space-charge limited with a fielddependent charge carrier mobility and that trapping in energetically distributed trap states is additionally involved at low voltages and especially for thick layers. However, in order to develop a consistent device model, the second important parameter, namely the temperature dependence of the current-voltage characteristics has to be investigated. But before performing the full numerical analysis of the temperature dependent $I-V$ characteristics we will first demonstrate that the TCLC model used before by several groups [81-83] quantitatively fails, because the field and temperature dependence of the charge carrier mobility is ignored, although it can qualitatively explain several features observed in the temperature dependence of the current.

Fig. 15(a) shows a set of data for the $300 \mathrm{~nm}$ thick device between 340 and $120 \mathrm{~K}$ in a doublelogarithmic plot. In this representation the curves seem to nicely follow power laws $j \propto V^{l+1}$ with increasing exponent $(l+1)$ for decreasing temperature. This behaviour is predicted in the TCLC model with an exponential trap distribution (and a field-independent mobility) and has been used to derive the characteristic trap energy $E_{t}$ from the temperature dependence of $l=E_{t} / k_{B} T$. According to this relation a plot of the temperature dependent exponent from the power laws in the $I-V$ characteristics vs. the reciprocal temperature should yield a straight line through the origin. The corresponding plot (Fig. 15(b)) shows indeed a linear dependence with a slope yielding a trap energy of about $0.16 \mathrm{eV}$ very similar to values reported earlier by other groups [81-83]. However, the straight line does not go through the origin. More severe is the fact that this model requires a mobility independent of the electric field to arrive at the used analytical power laws. However, this is not fulfilled for Alq (cf. section IV B). Moreover, we will show below that the field dependence of the mobility becomes increasingly more pronounced for lower temperatures so that there is also a strong effect of the mobility on $j(T)$.

Therefore, we have analyzed the temperature dependent $I-V$ characteristics of our $\mathrm{Al} / \mathrm{Alq} / \mathrm{Ca}$ devices using numerical simulations of SCLC with an exponential trap distribution $H_{t}(E)=$ $\left(N_{t} / E_{t}\right) \exp \left[\left(E-E_{C}\right) / E_{t}\right]$ and including a fielddependent mobility of the Poole-Frenkel type $\mu(F, T)=\mu_{0}(T) \exp [\beta(T) \sqrt{F}]$ with parameters $\mu_{0}$ and $\beta$ to be determined for each temperature. The analysis has been performed on three devices with thicknesses of about 100, 200 and $300 \mathrm{~nm}$ in the temperature range between 320 and 100K. Fig. 16(a) shows the experimental data of the $300 \mathrm{~nm}$ thick device together with numerically calculated $I-V$ curves using a trap density $N_{t}=$ $1.3 \times 10^{17} \mathrm{~cm}^{-3}$ and a value of $E_{t}=0.14 \mathrm{eV}$ characterizing the decay of the trap distribution with energy from the transport level into the gap. Additional material parameters in the simulation are the density of transport states $N_{C}=5 \times 10^{21} \mathrm{~cm}^{-3}$ (corresponding to the density of molecules) and the dielectric constant $\varepsilon=3.5$ of Alq. With these parameters the values of $\mu_{0}$ and $\beta$ have been adjusted at each temperature to obtain the best agreement between experiment and simulation. It is seen that reasonable agreement can be obtained for all temperatures, especially the characteristic curvature of the $I-V$ curves is well reproduced by the simulations. The resulting values of $\mu_{0}$ and $\beta$ are plotted in Fig. 16(b). There is a strong temperature dependence of $\mu_{0}$ typical of disordered molecular solids [34]. The analysis of such data with different models will be discussed in more detail in the following section IV B on transient electroluminescence. Here we anticipate that the temperature and field-dependent mobility can be described by the modified Poole-Frenkel equation (cf. Eq. 28) [89]:

$$
\begin{gathered}
\mu(F, T)=\mu_{P F} \exp \left(-\frac{\Delta E-\beta_{P F} \sqrt{F}}{k_{B} T_{\text {eff }}}\right) \\
\text { with } \frac{1}{T_{\text {eff }}}=\frac{1}{T}-\frac{1}{T_{0}} .
\end{gathered}
$$

According to this equation, from a plot of the fitted data of $\log \left(\mu_{0}\right)$ and $\beta$ vs. $1 / T$ one should obtain two linear dependencies from which it is possible to determine the parameters in the PooleFrenkel equation. From the data analysis between 320 and $180 \mathrm{~K}$ the following values have been obtained: $\mu_{P F}=1.62 \times 10^{-3} \mathrm{~cm}^{2} / \mathrm{Vs}, T_{0}=795 \mathrm{~K}$, $\Delta E=0.50 \mathrm{eV}$ and $\beta_{P F}=4.55 \times 10^{-23} \mathrm{~J}(\mathrm{~cm} / \mathrm{V})^{1 / 2}$. The deviations of $\log \left(\mu_{0}\right)$ and $\beta$ from the linear dependence on $T^{-1}$ below about $180 \mathrm{~K}$ indicate that at low temperature other transport mechanisms like e.g. variable range hopping or tunneling may come into play. These processes are known to have a weaker temperature dependence than a thermally activated one or can be even independent of temperature [90]. In section IV B we will present results on the temperature and fielddependent mobility in Alq from transient electroluminescence measurements, which have also been analyzed with this model. The parameters obtained there are comparable although they were achieved with $\mathrm{Alq}(2)$ from a different origin.

We emphasize that with these 6 parameters $(2$ for the trap distribution and 4 characterizing the charge carrier mobility) it is possible to achieve a good description of the temperature and thickness dependence of the $I-V$ characteristics. The latter has been demonstrated already in Fig. 14 for the room temperature data. This figure also 
nicely shows that trapping and the field-dependent charge carrier mobility come into play in different parameter ranges of the applied voltage and the device thickness. While trapping is important for thick layers at low voltage, the field dependence of the mobility is the dominant factor at high voltage, when traps become filled, or for thin layers, where the influence of traps is almost negligible (as we have noticed already in a previous publication [91]). Thus, the parameters for the traps and the mobility can be determined independently by focusing on different regions of the $I-V$ characteristics and by choosing suitable device thickness.

In spite of the good description of the $I-V$ characteristics with a hopping-type field and temperature dependent charge carrier mobility and an exponential distribution of traps states below the conduction level, some comments to the used trap distribution are appropriate. As discussed already in Refs. [5,94], it is difficult to distinguish between an exponential and a Gaussian trap distribution from the measurement of the $I-V$ characteristics alone. The reason is that the shift of the Fermi level, as the applied bias is varied, is usually too small to probe an energy range of the trap distribution large enough to make a unique distinction. What can be concluded from the temperature dependence of our data is that the trap distribution must have its maximum at or only a little below the transport level and that the relevant traps for the $I-V$ characteristics are shallow. A numerical analysis using a Gaussian distribution of trap states yields only marginally worse agreement between experiment and simulation. Thus, from this point of view, an exponential trap distribution is not necessarily the only possibility to describe the experiments. What is needed, is independent information about the energetic and spatial distribution of traps in the material. This issue has been addressed recently by measurements of thermally stimulated luminescence and currents on Alq [92,93]. Although traps were detected in a wide energy range up to $0.5 \mathrm{eV}$, it was not yet possible to reconstruct the trap distribution from these data. So, there is certainly more work needed to determine trap distributions in molecular organic films.

Another aspect is also important to mention, namely the influence of charge carrier injection. The fact that the $I-V$ characteristics can be sufficiently well described by SCLC with a field and temperature dependent mobility does not mean that the barrier for charge carrier injection is completely negligible. Looking at the thickness dependence of the current at constant field (Fig. 13) one can see that for thin layers (around 100nm) the dependence becomes weaker than $j \propto d^{-1}$, indicating an increasing influence of the injection barrier for thin layers. This means that the electric field at the injecting contact may no longer vanish as originally required for SCLC, nevertheless, it is still different from $F=\mathcal{V} / d$ as it were for pure injection limitation. Therefore, even for a finite but not too large injection barrier, the $I-V$ characteristics are still dominated by the presence of space-charges and will not change their shape significantly. The magnitude of the injection barrier primarily depends on the choice of the injecting contact. While Stößel et al. have recently demonstrated by transient SCLC that for thick Alq layers $(d>200 \mathrm{~nm})$ an ohmic contact for electron injection can be achieved using an ultra-thin $(0.1-0.2 \mathrm{~nm}) \mathrm{LiF}$ layer between Alq and an Al metal electrode [82], Barth et al. have found that using a $\mathrm{Mg}: \mathrm{Ag}$ cathode leads to RS-type injection limited behaviour [80]. On the other hand, as has been demonstrated by Wolf et al. there is an interdependence of the tolerable injection barrier to achieve SCLC and the mobility of the organic material [76]. Thus the differences in device characteristics reported in the literature may be partly due to different material and preparation conditions leading to variations in the mobility of Alq and different boundary conditions at the injecting contacts.

\section{Light-emitting single and hetero-layer devices}

In order to obtain light emission from organic films by charge carrier recombination one has to apply suitable contacts to achieve injection of both positive and negative carriers. When going from unipolar to bipolar currents two new phenomena become important, namely charge neutralization and recombination [35]. The former means that the space-charges of electrons and holes can neutralize each other to a large extent so that a doublecarrier current can be significantly higher than either single-carrier current. This effect can be even more pronounced if charge carrier trapping is involved. Recombination in organic LEDs has been found to be a bimolecular process following the Langevin theory $[6,37,38,48,49]$. As already mentioned in section II an analytic solution of the double-carrier transport equations is only possible under certain assumptions like a mobility independent of the electric field and the absence of traps. In the general case numerical solutions are required, which however necessitates detailed knowledge about single-carrier transport for both carrier types together with the boundary conditions for carrier injection at the contacts. At present we do not have enough information to perform such an analysis for our devices. Therefore we will only give a qualitative discussion of light-emitting Alq single-layer and NPB/Alq hetero-layer devices.

In Fig. 17 we show a comparison of current, luminance and EL quantum efficiency of ITO/Alq/Ca double-carrier devices made from different Alqs together with their single-carrier pendants with Al anodes. The respective Alq films were prepared in the same evaporation run on 
neighboring positions in the evaporator. Therefore the layer thicknesses and the Ca cathodes are identical for the corresponding unipolar and bipolar devices. Also the ITO anode treatment was the same for all double-carrier devices. As compared to the electron-only devices in all cases an enhancement of the current is seen, however by different factors. At an effective voltage $\mathcal{V}=6 \mathrm{~V}$ the enhancement factor is 5 for $\mathrm{Alq}(1), 15$ for $\mathrm{Alq}(2)$ and 300 for Alq(3). This behaviour is indicative of an increasing trap concentration from $\operatorname{Alq}(1)$ to $\operatorname{Alq}(3)$ as was already pointed out in the preceding discussion of single-carrier devices. An enhancement by two orders of magnitude is also predicted in numerical simulations of bipolar trap-charge limited conduction for an $l$-value of 7 [52]. This large difference between unipolar and bipolar currents in the presence of traps shows that it is problematic to derive the correct transport parameters from $I-V$ characteristics under conditions of double-carrier injection $[81,83]$.

The luminance-voltage curves show that the differences in the bipolar device currents between the materials is also reflected in the luminance, which is proportional to the current. In $\mathrm{Alq}(2)$ and $\mathrm{Alq}(3)$ with worse transport properties much higher voltages are required to achieve the same luminance values as with $\mathrm{Alq}(1)$. Looking at the external quantum efficiency, Alq(1) has the highest values of $\eta_{E L} \approx 0.5 \%$ being virtually independent of the applied voltage above $4 \mathrm{~V}$. The values of $\eta_{E L}$ are almost a factor of 2 lower for the other materials and especially $\mathrm{Alq}(3)$ shows a continuous increase of $\eta_{E L}$ with the applied voltage up to $10 \mathrm{~V}$. Since measurements of the photoluminescence quantum efficiency have, within the experimental error, yielded identical values of $\eta_{P L} \approx 20 \%$ for all three materials, the different absolute values and voltage dependence of $\eta_{E L}$ will largely result from the influence of carrier trapping on the recombination kinetics and the charge carrier balance. Owing to the short exciton diffusion length in most disordered organic films the influence of traps on the exciton lifetime is probably of minor importance.

A further improvement of device efficiency and light output can be achieved in hetero-layer devices combining the hole transporting NPB with Alq(1). Fig. 18 shows current- and luminancevoltage curves of hetero-layer devices with different cathode materials. A thickness of $60 \mathrm{~nm}$ for the Alq layer has been determined to yield the maximum quantum efficiency (in accordance with other work [95]), whereas a thickness variation of the NPB layer between 10 and 60nm had no measurable effect. It is seen that the external quantum efficiency for a Ca cathode shows a further increase as compared to the single-layer device and reaches values of about $2 \%$.

The external EL quantum efficiency is given as a product of several factors [96]:

$$
\eta_{E L}=\eta_{P L} \cdot \eta_{S} \cdot \gamma \cdot \eta_{o u t}
$$

with $\eta_{P L}$ the photoluminescence quantum efficiency, $\eta_{S}$ the efficiency for the production of singlet excitons, $\gamma$ the charge balance factor and $\eta_{\text {out }}$ the extraction efficiency. The definition of the charge balance factor is

$$
\gamma=j_{r} / j
$$

with the recombination current

$$
j_{r}=j_{h}-j_{h}^{\prime}=j_{e}-j_{e}^{\prime}
$$

and the total current in the external circuit

$$
j=j_{h}+j_{e}^{\prime}=j_{e}+j_{h}^{\prime}
$$

$\left(j_{e, h}\right.$ are the injected electron and hole currents, respectively, and the primed quantities denote the respective fractions reaching the counter electrode without recombining.) With $\eta_{S}=0.25$ from spin statistics and the measured value of $\eta_{P L} \approx 0.2$ it follows that the product $\gamma \cdot \eta_{\text {out }}$ must be about 0.4 to yield $\eta_{E L}=0.02$. Since the value of $\eta_{E L}$ has been derived from the measurement of the total light output in an integrating sphere (cf. section III) in our case $\eta_{\text {out }}$ will be certainly larger than the usual value of $1 /\left(2 n^{2}\right) \approx 1 / 6$ [97] ( $n$ being the refractive index of the emissive medium). Thus we can conclude that the charge balance factor $\gamma$ is close to unity. According to Eqs. (23-25) this is achieved if both $j_{h}^{\prime}$ and $j_{e}^{\prime}$ vanish. Therefore, the increase of the quantum efficiency in the heterolayer device is mainly due to the excellent electron blocking properties of NPB (see also section IV B). Other factors are the shift of the recombination zone away from the ITO contact, where luminescence quenching could happen, and the higher supply of holes from an NPB layer into Alq as compared to an ITO contact with a much larger energy barrier (see Fig. 5).

The comparison of different cathode materials in Fig. 18 shows that still a slight increase of current, luminance and quantum efficiency can be achieved by using a $\mathrm{LiF} / \mathrm{Al}$ cathode instead of Ca. This means that the electron injection barrier from $\mathrm{Ca}$ into Alq is not negligible, at least in a hetero-layer device. This is not necessarily in contradiction to the fact that $\mathrm{Al} / \mathrm{Alq} / \mathrm{Ca}$ single-layer electron-only devices with thickness between 100 and $300 \mathrm{~nm}$ have revealed SCLC, since both the reduction of the layer thickness and double-carrier injection with the concomitant space-charge neutralization increase the demand for carriers to be supplied by a contact. Thus a contact which is found to be ohmic for unipolar currents may become more injection limited under bipolar current flow. 


\section{B. Charge carrier mobilities in Alq}

\section{Field and temperature dependence of the electron mobility in Alq}

We have demonstrated in the preceding section that the field and temperature dependent charge carrier mobility in Alq plays an important role and determines the current-voltage characteristics at higher voltages. Thus it is desirable to directly determine carrier mobilities and compare the data with the results from simulations of the $I-V$ characteristics.

Because of their use as organic photoconductors in xerography a huge amount of data mostly determined by the time-of-flight (TOF) technique can be found for the hole conducting aromatic amines, like TPD and NPB or derivatives [34,98]. The room temperature hole mobilities in neat films of TPD or NPB are found to be comparatively high with values around $10^{-3} \mathrm{~cm}^{2} / \mathrm{Vs}$ and display a very weak dependence on the electric field (see Fig. 6). The more challenging and also the limiting material in a hetero-layer device is Alq. In spite of the enormous progress in the development of OLEDs there are still comparatively few data on carrier mobilities in Alq, especially since all existing studies were performed at room temperature $[54,55,74,84,99,100]$. From the TOF data shown in Fig. 6 it is evident that for electrons in Alq the zero field mobility is much lower and the field dependence much stronger than for holes in TPD. This is a consequence of the dispersive nature of the electron transport in Alq, which strictly speaking makes the definition of a microscopic mobility impossible. As a consequence the macroscopically obtained values depend on experimental parameters like the thickness of the sample [34]. This circumstance makes the usage of data determined by the TOF method problematic since the usual layer thickness for these measurements of several $\mu \mathrm{m}$ is about two orders of magnitude larger than the typical thickness in OLEDs. For this reason additional information on carrier mobilities in OLEDs is needed for a comparison with the results of device simulations. Moreover, so far there were no reports on the temperature dependence of the charge carrier mobility in Alq. Therefore we used timeresolved transient electroluminescence as an independent experimental technique to obtain charge carrier mobilities directly on OLED structures, as has been done before by other groups on different materials [101-105].

In principle one measures the time-resolved EL response to a pulse excitation, usually a rectangular voltage pulse. The charge carrier mobility can be obtained from the delay time between the application of the voltage pulse and the first appearance of EL, which marks the arrival of carriers at the recombination zone. At a given sample thickness $d$ the delay time $t_{d}$ depends on the mobility $\mu$ as:

$$
t_{d}=\frac{d}{\mu F}
$$

In spite of the simplicity of the experiment, the correct assignment of the observed time lag in EL to charge carrier mobilities is not straightforward. First, the experiment probes the mobility of carriers of both sign $\left(\mu=\mu_{h}+\mu_{e}\right)$. Only if the mobility of one species is much higher this carrier type will dominate the signal. Then, one needs information about the location and width of the recombination zone, which is again simplified if one carrier type is much faster. Furthermore one has to take into account luminescence quenching in the vicinity of metals if the recombination zone is located at an injecting contact $[48,106-108]$, which is the case for most single-layer OLEDs. And finally, in multi-layer devices the accumulation of charges at internal interfaces and the concomitant redistribution of the electric field can also have an influence on charge carrier injection and transport through the device $[109,110]$.

Hosokawa et al. were the first to use transient EL for the determination of the electron mobility in Alq in a TPD/Alq hetero structure device at room temperature [101]. In a subsequent work [111] they were able to extend the existing range of data for the electron mobility in Alq to higher fields than available by TOF $[54,55]$. However, they did not take into account the presence of a built-in voltage and of an inhomogeneous field distribution inside the device, which becomes important as will be seen below.

We have performed transient EL measurements on a simple hetero-layer device consisting of TPD and $\mathrm{Alq}(2)$, each $60 \mathrm{~nm}$ thick, sandwiched between ITO and Ca electrodes. In this structure the recombination zone is located at the TPD/Alq interface. Additionally, as will be seen in the following section (IV C) the voltage drop under forward bias occurs almost completely at the Alq layer. Then with the large difference in mobilities $\left(\mu_{h, \mathrm{TPD}} \gg \mu_{e, \mathrm{Alq}}\right)$ the transient response will be dominated by the transport of electrons through the Alq layer. Hence, their mobility is given by:

$$
\mu_{e, \mathrm{Alq}}=\frac{d_{A l q}}{t_{d} F} \quad \text { with } \quad F=\frac{V-V_{b i}}{d_{\mathrm{Alq}}}
$$

Again, for the calculation of the effective field inside the organic layers, the built-in voltage has to be considered. A typical example of a transient EL signal with the delay indicated by an arrow is shown in Fig. 19. The time lag in transient EL has been investigated for different pulse amplitude and temperature yielding the values of the field and temperature dependent electron mobility in Alq displayed in Fig. 20. At all temperatures the data display a field dependence according to $\mu(F)=$ $\mu_{0} \exp (\beta \sqrt{F})$ with decreasing zero field mobility $\mu_{0}$ and increasing $\beta$-factor for lower temperature. This behaviour is typical of hopping transport in 
disordered organic solids and has been analyzed using either a modified Poole-Frenkel model (PFM) [89] or a Gaussian disorder model (GDM) [112], the latter was recently extended to include correlation effects [113-115]. Since our data set is certainly too limited to distinguish whether one or the other model is more appropriate to describe transport in Alq we will only use the two most popular ones.

In the Poole-Frenkel model the mobility can be described as a field and temperature assisted detrapping process of a carrier from the Coulomb potential of a charged trap. The mobility is then given by [89]:

$$
\begin{gathered}
\mu(F, T)=\mu_{P F} \exp \left(-\frac{\Delta E-\beta_{P F} \sqrt{F}}{k_{B} T_{\text {eff }}}\right) \\
\text { with } \frac{1}{T_{\text {eff }}}=\frac{1}{T}-\frac{1}{T_{0}},
\end{gathered}
$$

where $\Delta E$ is the activation energy at zero electric field, $T_{0}$ an empirical parameter and $\mu_{P F}$ the mobility at $T=T_{0}$. The Poole-Frenkel factor is related to the dielectric constant by

$$
\beta_{P F}=\sqrt{\frac{q^{3}}{\pi \varepsilon \varepsilon_{0}}} .
$$

The parameters of the PFM can be determined from the temperature dependent values of $\mu_{0}$ and $\beta$ by plotting $\log \left(\mu_{0}\right)$ and $\beta$ vs. $T^{-1}$. Both should follow linear dependencies if the PFM is adequate. This is approximately fulfilled between room temperature and $200 \mathrm{~K}$ and the obtained parameters are: $\mu_{P F}=1.2 \times 10^{-4} \mathrm{~cm}^{2} / \mathrm{Vs}, T_{0}=430 \mathrm{~K}, \Delta E=$ $0.43 \mathrm{eV}$ and $\beta_{P F}=4.1 \times 10^{-23} \mathrm{~J}(\mathrm{~cm} / \mathrm{V})^{1 / 2}$. Fig. 20 compares the experimental mobility data with the analysis by the PFM.

Another widely used expression is the Gaussian disorder model [112]:

$$
\begin{aligned}
\mu(F, T) & =\mu_{\infty} \exp \left[-\left(\frac{2 \sigma}{3 k_{B} T}\right)^{2}\right] . \\
& \cdot \exp \left\{C\left[\left(\frac{\sigma}{k_{B} T}\right)^{2}-\Sigma^{2}\right] \sqrt{F}\right\}
\end{aligned}
$$

This equation was derived from Monte Carlo simulations of the hopping processes of charge carriers in a material with energetic $(\sigma)$ and positional $(\Sigma)$ disorder described by Gaussian distribution functions. $\mu_{\infty}$ is the high temperature limit of the mobility and $C$ a specific parameter which is obtained from the simulations as $C=2.9 \times 10^{-4}(\mathrm{~cm} / \mathrm{V})^{1 / 2}$. Again the parameters of the GDM can be determined from a linear dependence of $\log \left(\mu_{0}\right)$ and $\beta$ in a plot vs. $T^{-2}$. The obtained parameters are: $\mu_{\infty}=$ $5.2 \times 10^{-4} \mathrm{~cm}^{2} / \mathrm{Vs}, C=2.7 \times 10^{-4}(\mathrm{~cm} / \mathrm{V})^{1 / 2}$, $\Sigma=1.8$ and $\sigma=100 \mathrm{meV}$. As indicated in Fig. 20 the data between room temperature and $200 \mathrm{~K}$ can thus also be fitted using the GDM.
It is obvious that within the scattering of experimental data both models are in agreement with the measurements above 200K. Experimentally, the main difference between them (apart from the different mechanisms behind them) is the temperature dependence. However, there are very rare examples where a clear distinction between the two models has been possible based on the temperature dependence [116]. In our case, it seems that the description with the PFM is better at low temperature. However, one should note that the transients at low temperature are relatively noisy and can be influenced by trapped charges remaining in the sample from the preceding pulse. Nevertheless, one can compare the results with other work in the literature. Blom et al. have used the PFM to analyze their results of the hole mobility in polymer LEDs based on a soluble PPV derivative [117,118] and obtained values of $\mu_{P F}=3.5 \times 10^{-7} \mathrm{~cm}^{2} / \mathrm{Vs}, T_{0}=600 \mathrm{~K}$, $\Delta E=0.48 \mathrm{eV}$ and $\beta_{P F}=4.6 \times 10^{-23} \mathrm{~J}(\mathrm{~cm} / \mathrm{V})^{1 / 2}$, which are - apart from the prefactor $\mu_{P F}$ - comparable to the ones obtained here. The GDM has also been widely used to describe charge carrier mobility in disordered molecular solids [34,119]. The disorder parameter $\sigma \approx 100 \mathrm{meV}$ in Alq is considerably larger than in TPD or NPB $(\sigma \approx 80 \mathrm{meV})$ $[34,72]$ which can be one reason for the stronger field dependence of the mobility in Alq.

From this comparison one can conclude that the electron transport in Alq shows the typical features of charge transport in disordered organic films. The important point in the context of this paper, however, is the fact that the electron mobility in Alq is strongly field and temperature dependent, which has to be taken into account in the modeling of the $I-V$ characteristics. The direct comparison of the results obtained within the PFM from $I-V$ characteristics and transient EL shows acceptable agreement for the parameters although both experiments were performed on different materials. Apart from this experimental issue, another reason for a possible discrepancy can be sought in the method of analyzing the transient EL response, since the delay time until the onset of EL corresponds to the arrival of the fastest carriers at the recombination zone rather than that of the majority of the carriers. Other authors have alternatively used the rise time of the EL signal necessary to reach a stationary light output [109] or an extrapolation of the time integrated EL signal towards zero [27]. It can be seen qualitatively from Fig. 19 that this will certainly lead to lower mobility values so that one can expect changes in the parameters of the models, however, the general behaviour will not be affected significantly. 
Following early time-of-flight measurements by Kepler et al. [54] Alq was widely regarded as a purely electron conducting material with negligible hole drift mobility, since the obtained values at low fields were almost two orders of magnitude lower than the electron mobility. On the other hand, TOF experiments by Lin et al. on multi-layer structures with only a few nm thick Alq films have indicated that the hole drift mobility in Alq may be comparable to the values for electrons [100]. Thus it was not clear whether the hole transport in Alq films with the typical thickness used in OLEDs can be neglected especially at higher electric fields.

Therefore we measured the hole drift mobility in Alq using a double hetero structure device (see inset in Fig. 21) consisting of two TPD/Alq double layers. The top Alq layer served as electron injection and emission layer. To identify the recombination zone this Alq layer was partially doped with $2 \%$ of a red fluorescent laser dye (DCM2). The 50nm thick TPD layer below had the function of a buffer layer to block electrons injected at the cathode from reaching the bottom Alq layer through which the hole transport was to be probed. The Alq thickness was varied from $50 \mathrm{~nm}$ to $200 \mathrm{~nm}$ to verify that the measured delay time was determined by the hole drift mobility in this layer. Finally the $10 \mathrm{~nm}$ thick TPD layer at the ITO anode merely served as injection layer for holes into Alq.

Measurements of luminance-voltage characteristics showed detectable light output above a voltage of approximately $4 \mathrm{~V}$ (see Fig. 21). Thus, one has to conclude that either holes are transported through the Alq layer sandwiched between the two TPD layers or electrons through the $50 \mathrm{~nm}$ thick TPD layer. However, the emission spectrum (see inset in Fig. 21) gives unique evidence that charge carrier recombination occurs only in the dye doped Alq layer at the top, because no EL intensity was measured below wavelengths of 520nm where one would expect the maximum of the Alq emission. In a control experiment with the bottom Alq layer doped with DCM2 green emission originating again from the now undoped top Alq layer was obtained [125]. This proves that the electron current through TPD can be indeed neglected as compared to the hole current through Alq, and it also rules out the possibility that trapping of electrons at DCM2 molecules is the dominant factor which determines the location of the emission zone in the device.

From TOF measurements the drift mobilities of holes in TPD and electrons in Alq are supposed to be higher by orders of magnitude than the hole drift mobility in Alq. For this reason the transit time of electrons in the top Alq layer and the transit time of the holes through both TPD layers can be neglected. For the calculation of the electric field within the bottom Alq layer we assume that the applied voltage drops homogeneously over this Alq layer and the 50nm thick TPD layer. With the thickness of the bottom Alq layer $d_{\mathrm{Alq}}$ (varied from $50 \mathrm{~nm}$ to $200 \mathrm{~nm}$ ) and the TPD layer of thickness $d_{\text {TPD }}(=50 \mathrm{~nm})$ we obtain an electric field of

$$
F=\frac{V-V_{b i}}{d_{\mathrm{Alq}}+d_{\mathrm{TPD}}} .
$$

With respect to the higher drift mobilities of electrons in Alq and holes in TPD this estimation is reasonable as the time for the injected charge carriers to traverse the first organic layer can be neglected. As a consequence the space charges within both organic layers next to the electrodes are built up immediately leading to a negligible voltage drop over these layers. Then the hole drift mobility in Alq can be calculated as

$$
\mu_{h, \mathrm{Alq}}=\frac{d_{\mathrm{Alq}}}{t_{d} F} .
$$

Fig. 22 shows the hole mobility in Alq obtained on the double hetero structure at room temperature for the three different Alq layer thicknesses. From the linear fit we obtain a dependence of the hole drift mobility on the electric field as $\mu_{h, \mathrm{Alq}}=$ $6 \times 10^{-11} \mathrm{~cm}^{2} / \mathrm{Vs} \cdot \exp \left(9 \times 10^{-3}(\mathrm{~cm} / \mathrm{V})^{1 / 2} \cdot \sqrt{\mathrm{F}}\right)$. These results are in excellent agreement with results of Kepler et al. and Naka et al. who measured the field-dependent hole mobility in about $10 \mu \mathrm{m}$ thick Alq films using the time-of-flight method $[54,84]$. This agreement between our data and the TOF data obtained on samples with two orders of magnitude difference in thickness is astonishing. It can be taken as an indication for non-dispersive hole transport in Alq. Naka et al. recently also arrived at this conclusion, because their photocurrent transients had a shape typical for non-dispersive transport with a clearly visible transit time on a linear time scale. Thus, Alq has the unusual property that transport for the carrier species with the much higher mobility (electrons) is dispersive while it is non-dispersive for the lower mobility carriers (holes).

The comparison of the electron and hole drift mobility in Alq at $300 \mathrm{~K}$ (Fig. 22) shows that the hole mobility at low fields is lower by almost three orders of magnitude. However, with increasing electric field the difference of the mobilities of both charge carrier types becomes smaller due to the stronger dependence of the hole drift mobility on the electric field. From this point of view the hole transport in Alq can not generally be neglected in organic LEDs.

\section{Interfacial charges and electric field distribution in hetero-layer devices}

The measurement of the bias and frequency dependent device capacitance is a well-established technique for the investigation of conductivity, 
doping and trap states in organic semiconductors. For a number of conjugated polymers this method has been used to prove the existence of p-type doping leading to Schottky junctions with low work function metals $[23,120,121]$. In combination with temperature dependent measurements the energy of shallow acceptor states and deep trap states has been determined $[25,122]$.

Here we have used this technique to obtain information on interfacial charges and the electric field distribution in hetero-layer devices comprising NPB as hole transport layer and Alq as electron transport and emissive material. For this type of hetero-layer devices it has been found already from the analysis of current-voltage characteristics by Matsumura et al. [123] and from electroabsorption spectroscopy by Rohlfing et al. [124] that under forward bias the voltage drop at the Alq layer is much larger than at the NPB layer. This is not unexpected since an accumulation of injected holes at the NPB/Alq interface will occur due to the energy barrier for holes (see Fig. 5) and also since the hole mobility in NPB is much larger than in Alq. In the following, we will show that impedance spectroscopy can yield information about the potential distribution in hetero-layer devices under reverse bias where other techniques fail.

Typical data of the complex impedance $\hat{Z}=$ $Z^{\prime}+i Z^{\prime \prime}=|\hat{Z}| \cdot e^{i \varphi}$ are shown in Fig. 23 for a hetero-layer device with $60 \mathrm{~nm}$ NPB and $60 \mathrm{~nm}$ Alq. For large reverse bias the device behaves essentially like an insulating dielectric with a purely capacitive response $(\varphi=-\pi / 2)$. Above a turnon voltage in the range 2 to $2.5 \mathrm{~V}$ the phase $\varphi$ increases from $-\pi / 2$ to 0 indicating conducting behaviour and the modulus of the impedance $|\hat{Z}|$ also decreases rapidly with increasing voltage. As discussed before this turn-on voltage can be approximately identified with the built-in voltage $V_{b i}$ corresponding to the contact potential difference of the ITO and Ca electrodes, which has to be overcome before a non-negligible drift current can flow. Below the built-in voltage an additional feature appears at about $-0.5 \mathrm{~V}$ : A weak bump occurs in the phase and $|\hat{Z}|$ changes by a factor of 5 . This feature has no equivalent in the $I-V$ characteristics. The $I-V$ characteristics of NPB/Alq heterolayer devices under reverse bias are qualitatively similar to the in detail discussed characteristics of single-layer devices (see section IV A). The current below $V_{b i}$ is usually very low $\left(10^{-8} \mathrm{~A} / \mathrm{cm}^{2}\right.$ or less) and varies almost linearly with the applied voltage between $V_{b i}$ and large negative values down to $-10 \mathrm{~V}$ without showing any additional structure. Thus the fact that the above mentioned feature under reverse bias is only observed for an a.c. stimulus indicates that an internal redistribution of the electric field instead of a change of the total ohmic device resistance is probably the origin.

We have investigated the bias-dependent impedance below $V_{b i}$ in detail by systematically varying the thickness of the NPB and the Alq layer independently. In the following, we will only discuss the differential capacitance

$$
C=\frac{\mathrm{d} Q}{\mathrm{~d} V}=(1 / 2 \pi f) \cdot\left(-Z^{\prime \prime}\right) /\left(Z^{\prime 2}+Z^{\prime 2}\right),
$$

which - although being only part of a full impedance analysis - allows to draw the important conclusions required here.

Fig. 24 shows two groups of data for devices with 60 and 120nm Alq layer thickness and NPB thicknesses varying from 0 to $60 \mathrm{~nm}$. In both data sets two different voltage regimes separated by a transition voltage $V_{0}$ (indicated by the arrows) can be identified. For voltages well below $V_{0}$ the measured value of the capacitance depends on the overall thickness of both organic layers and is given by $C_{\mathrm{tot}}=\left(C_{\mathrm{NPB}}^{-1}+C_{\mathrm{Alq}}^{-1}\right)^{-1}$ as expected for two dielectrics in series. Above $V_{0}$ the capacitance increases and reaches at the built-in voltage a value equivalent to the capacitance of the Alq layer alone: $C_{\text {tot }}=C_{\text {Alq }}$. Using the geometrical relationship $C=\varepsilon \varepsilon_{0} A / d$ with the device area $A$ and equal dielectric constants $\varepsilon \approx 3.5$ for both NPB and Alq, it can be readily verified that for $V \ll V_{0}$ the value of $d$ corresponds to the total organic layer thickness, whereas for $V \approx V_{b i}$ only the thickness of the Alq layer is relevant.

It is remarkable that the transition does not occur at the built-in voltage, but at a voltage $V_{0}$ which is lower than $V_{b i}$ and even negative. The steep increase of the capacitance around $V_{0}$ occurs over a range of about $1 \mathrm{~V}$, which is larger than the oscillator amplitude and not influenced by it, as long as the a.c. amplitude is kept below $0.5 \mathrm{~V}$. It is evident from Fig. 24 that the transition voltage $V_{0}$ is independent of the NPB layer thickness, however, $V_{0}$ is significantly different for Alq thicknesses of 60 and $120 \mathrm{~nm}$. Fig. 25 shows in more detail the dependence of $V_{0}$ on the Alq thickness. Here the thickness of the NPB layer was kept constant at $60 \mathrm{~nm}$ and the Alq thickness varied from 10 to $240 \mathrm{~nm}$. All curves show the above described increase from a lower capacitance value at large reverse bias, corresponding to the total organic layer thickness, to a higher value given by the Alq thickness. The voltage, where the transition occurs, decisively depends on the thickness of the Alq layer. Obviously, the transition voltage is close to the built-in voltage for the thinnest Alq layer and shifts towards zero and negative voltage with increasing Alq thickness. The inset of Fig. 25 displays the dependence of the transition voltage $V_{0}$ (taken as the inflection point of the $C-V$ curves) on the Alq thickness. Within the error limits given by the width of the transition and the thickness uncertainty, the transition voltage depends linearly on the Alq thickness. The fitted straight line intersects the voltage axis at $(2.2 \pm 0.15) \mathrm{V}$, which is very close to the built-in voltage estimated from current-voltage characteristics, and its slope is $(-0.35 \pm 0.03) \mathrm{MV} / \mathrm{cm}$. 
For an explanation of the experimental observations listed above it is useful to consider the properties of the equivalent circuit of a hetero-layer device shown in Fig. 26. Each organic layer is represented by a parallel circuit of a capacitor and a resistor connected in series. An additional series resistor for the lead resistance of the electrodes has been neglected in this picture. In the absence of doping the values of the capacitance for each layer are determined by the geometry and the dielectric constant: $C_{i}=\varepsilon_{i} \varepsilon_{0} A / d_{i}(i=\mathrm{NPB}, \mathrm{Alq})$. They are independent of the applied bias since the dielectric constant is a bias-independent material constant. The resistance values are given by the conductivity and the geometry: $R_{i}=\sigma_{i} d_{i} / A$. The important point is now that the resistance is a strongly biasdependent quantity, since the value of $\sigma_{i}=q n_{i} \mu_{i}$ changes when the carrier density $n_{i}$ is increased by carrier injection. This happens when the voltage across an organic layer exceeds the flat-band voltage, which is equivalent to the built-in voltage in a single-layer device (cf. section IV A).

In general, the totally measured capacitance of such a double $R C$ network depends on the values of all its elements and the measurement frequency (for a detailed discussion see e.g. Ref. [121]). However, there are two limiting cases of interest here. One is that both the resistances, $R_{\mathrm{NPB}}$ and $R_{\mathrm{Alq}}$, are very large such that at a given measurement frequency $\omega=2 \pi f$ the condition $\omega \tau_{i} \gg 1$ with $\tau_{i}=R_{i} C_{i}$ is fulfilled. Then the total capacitance of the circuit is just the series sum of the two individual capacitances:

$$
\omega \tau_{i} \gg 1 \Rightarrow C_{\mathrm{tot}}=\left(C_{\mathrm{NPB}}^{-1}+C_{\mathrm{Alq}}^{-1}\right)^{-1} .
$$

This is the case for large reverse bias, where we know from the $I-V$ characteristics that the resistance of a single-layer device can be as high as $10^{12} \Omega$. With typical values of $C_{i} \approx 2 \mathrm{nF}$ the condition $\omega \tau_{i} \gg 1$ then holds for all practical measurement frequencies. The second interesting limit is when one resistance, here $R_{\mathrm{NPB}}$, is much smaller than the other one and at the same time the measurement frequency is not too high such that $\omega \tau_{\mathrm{NPB}} \ll 1$ holds. Then the measured total capacitance is just the capacitance of the Alq layer:

$$
R_{\mathrm{NPB}} \ll R_{\mathrm{Alq}} \text { and } \omega \tau_{\mathrm{NPB}} \ll 1 \Rightarrow C_{\text {tot }}=C_{\mathrm{Alq}} .
$$

In this case the measured capacitance is frequencydependent showing a dielectric relaxation step from the higher value $C_{\text {tot }}=C_{\mathrm{Alq}}$ at low frequency to the lower value $C_{\text {tot }}=\left(C_{\mathrm{NPB}}^{-1}+C_{\mathrm{Alq}}^{-1}\right)^{-1}$ above the relaxation frequency $\omega_{r}=\tau_{r}^{-1}$ with $\tau_{r}=R_{\mathrm{NPB}}\left(C_{\mathrm{NPB}}+C_{\mathrm{Alq}}\right)$ [121]. Such a frequency dependence of the capacitance is indeed observed experimentally as shown for two different bias values in Fig. 27. When the bias is below the transition voltage $V_{0}$ at the given Alq thickness (here
$\left.V=-1 \mathrm{~V}<V_{0} \approx-0.5 \mathrm{~V}\right)$ a frequency independent capacitance is found up to a few hundred $\mathrm{kHz}$, where the influence of the leads becomes important, and the value of the capacitance is equal to the series sum of both capacitances. On the other hand, when the bias is between $V_{0}$ and $V_{b i}$ (here $V=1 \mathrm{~V}$ ) then for frequencies sufficiently far below the relaxation frequency of about $50 \mathrm{kHz}$ the capacitance is equal to $C_{\text {Alq }}$ before it drops again to the value $C_{\text {tot }}=\left(C_{\mathrm{NPB}}^{-1}+C_{\mathrm{Alq}}^{-1}\right)^{-1}$. From the relaxation frequency the value of the NPB resistance can be determined as $360 \Omega$ which is orders of magnitude lower than for reverse bias. Fig. 27 also includes fit curves using values of $R_{\mathrm{Alq}}=10^{8} \Omega, C_{\mathrm{NPB}}=4.2 \mathrm{nF}$ and $C_{\mathrm{Alq}}=3.8 \mathrm{nF}$ together with $R_{\mathrm{NPB}}=10^{8} \Omega$ (for $V<V_{0}$ ) and $R_{\mathrm{NPB}}=360 \Omega$ (for $V>V_{0}$ ), respectively. To obtain a satisfactory fit above $2 \times 10^{5} \mathrm{~Hz}$ a serial lead resistance of $35 \Omega$ (e.g. for ITO) has been included.

Thus the observation of two bias regimes with different overall capacitance can be explained by a drastic drop of the resistance of the NPB layer at the critical voltage $V_{0}$. This means that at the voltage $V_{0}$ (which is still below $V_{b i}$ ) the NPB layer must have reached the flat band condition so that holes can be injected into NPB from the ITO anode. However, this in turn implies that the applied voltage now completely drops at the Alq layer. Therefore the internal electric field is zero in the NPB layer and equal to $\left(V_{0}-V_{b i}\right) / d_{\mathrm{Alq}}$ in the Alq layer. Following from Poisson's law such a discontinuity $\Delta F$ of the electric field at an interface can only be caused by an interfacial charge $Q_{0}$ :

$$
\Delta F=\frac{V_{0}-V_{b i}}{d_{\mathrm{Alq}}}=\frac{Q_{0}}{\varepsilon \varepsilon_{0} A}
$$

If this effect is an intrinsic property of the NPB/Alq interface, then the magnitude of $Q_{0}$ should not depend on external parameters, in particular not on the thickness of the organic layers. This in turn implies that the transition voltage $V_{0}$ should display a linear dependence on the Alq thickness, which is indeed seen in Fig. 25. From the slope $\Delta F=-0.35 \mathrm{MV} / \mathrm{cm}$ one directly obtains the magnitude of the interfacial charge density as $\sigma_{0}=Q_{0} / q A=\Delta F \varepsilon \varepsilon_{0} / q=-6.8 \times 10^{11} \mathrm{~cm}^{-2}$. So the physical reason behind the observed behaviour of the capacitance is the presence of negative interfacial charges at the NPB/Alq interface under reverse bias.

With the presence of these interfacial charges one can now draw a schematic view of the potential and field distribution inside a hetero-layer device under different bias conditions as shown in Fig. 28. For large negative bias the interfacial charge density is constant with the value of $\sigma_{0}$ given above. Although the electric field is discontinuous at the interface (see Fig. 28(a)), in the capacitance measurement one does not notice this because both organic layers are reverse biased and therefore have such a large resistance that one is always in the 
limit where the capacitance is the series sum of both layers. When the applied voltage approaches $V_{0}$ the NPB layer reaches the flat band condition (Fig. 28(b)) and its resistance drops drastically leading to the increase of the measured capacitance to the value $C_{\text {Alq }}$. For $V_{0}<V<V_{b i}$ it would be in principle possible that the interfacial charge density is constant. However, if this were the case, one would have different sign of the electric field in both organic layers and the electric potential would show a maximum at the interface. Furthermore, at $V=V_{b i}$ the flat band condition would not be achieved in both layers and the Alq layer would still be reverse biased. This is in clear contradiction to the strong increase of the current immediately above $V_{b i} \approx 2 \mathrm{~V}$. Therefore, it is reasonable to assume that for $V>V_{0}$, as soon as the flat band condition in NPB is attained, holes can be injected at the anode and pass through the NPB layer to reach the interface. There they will partly compensate the negative interfacial charges, so that the NPB layer stays in the flat band condition (see Fig. 28(c)). Thereby with increasing voltage the initial interfacial charge density $\sigma_{0}$ will be gradually reduced until at $V_{b i}$ it is fully neutralized and both the NPB and Alq layer have flat bands (see Fig. 28(d)). We note that the presence of excess charge carriers accumulated in the device to compensate the negative interfacial charges for $V_{0}<V<V_{b i}$ has been independently found by discharge measurements in this voltage range [110]. Finally, above $V_{b i}$ the device is forward biased and double-carrier injection occurs. Due to the strong difference in charge carrier mobility between NPB and Alq $\left(\mu_{h, \mathrm{NPB}} \gg \mu_{e, \mathrm{Alq}} \gg \mu_{h, \mathrm{Alq}}\right)[55,84,125]$ the voltage drop at the Alq layer will be still much higher than at the NPB layer (see for example Ref. $[123,124])$, but now its origin is an accumulation of injected positive carriers at the interface (see Fig. $28(\mathrm{e}))$.

Thus the assumption of negative interfacial charges at the NPB/Alq interface which are constant for $V \ll V_{0}$ and become gradually compensated for $V_{0}<V<V_{b i}$ is inevitable to explain the results of the capacitance-voltage measurements. Numerical simulations show that the observed thickness, bias and frequency dependence of the capacitance can almost quantitatively be described by assuming that a $1 \mathrm{~nm}$ thick part of the Alq layer directly at the interface contains acceptor like traps with a density of about $4 \times 10^{16} \mathrm{~cm}^{-3}$ [126]. At present, there is little direct information about the microscopic origin of these interfacial charges. It is an intrinsic property of the $\mathrm{NPB} /$ Alq interface, which is not dependent on external parameters like the layer thickness, the electrode material or the transport properties of Alq (hetero-layer devices with Alq(1) and Alq(2) give the same results in the bias and frequency dependent capacitance). Even if an additional $\mathrm{Cu}^{-}$ phthalocyanine layer is inserted between ITO and
NPB the transition voltage for a given Alq thickness is not changed [110]. However, preliminary experiments have shown that if an oxadiazole is used instead of Alq the magnitude of $\sigma_{0}$ increases.

Besides interface traps an alternative explanation would be to assume a partial charge transfer between the hole and electron conducting material in the hetero-layer device, the magnitude of which should depend on the difference of the redox potentials of the materials in the solid state. Such an effect was observed by photoelectron spectroscopy by Rajagopal et al. [31], who interpreted the shift of vacuum level positions at the NPB/Alq interface by a partial electron transfer from Alq to NPB. However, such a charge transfer would lead to a dipole layer without net charge [30]. Only if one type of charges in the dipole layer is able to move away from the interface or can be compensated by charges of the opposite sign in the respective organic layer, a net charge will remain. Nevertheless, one has to be aware that the comparison of impedance spectroscopy and photoelectron spectroscopy is problematic. While the latter is performed on the surfaces of in-situ grown organic films without electrodes, the former measures on a complete device, in which the behaviour is determined by several factors, such as the organic materials, the electrodes and the external bias conditions. Thus, further investigations with independent experimental techniques will be necessary to get more insight into the microscopic nature of interfaces in organic hetero-layer LEDs.

\section{CONCLUSIONS}

Organic light-emitting devices based on molecular materials are typically multi-layer structures incorporating interfaces between organic materials with different transport properties and energy levels. The understanding and quantitative description of the electrical behaviour and the light emission from these structures requires detailed knowledge of the properties of all its constituents. Here we have given a review on electrical transport in single- and hetero-layer devices based on aromatic amines like TPD or NPB and the aluminium chelate complex Alq. The combination of these two materials can be considered as the simplest kind of multi-layer device, which is still widely used in OLEDs. By separately studying singlecarrier single-layer devices of each material, we have demonstrated that the identification of the dominant current limiting mechanism is possible. We have shown that especially the thickness dependence of the current provides a unique criterion to distinguish between different limiting cases, even in the presence of a strongly field-dependent charge carrier mobility, where an analysis based on the functional dependence of the current on the voltage alone is not unambiguous. By comparing 
experimental results with numerical simulations a hopping-type field and temperature dependent charge carrier mobility in Alq could be identified as the main limiting factor. The additional influence of traps is clearly seen at low voltage and for thick layers, however, the underlying trap distribution has to be investigated further. Moreover, we have shown that important information about interfaces can be obtained for this kind of hetero structure from the reverse bias dependence of the capacitance. The reverse bias regime is also the region where the influence of deep traps can be seen. In the end, consistent device models or simulations will have to describe both the forward and reverse bias behaviour.

\section{ACKNOWLEDGMENT}

We thank M. Schwoerer, W. Rieß, G. Paasch, P.H. Nguyen, and S. Scheinert for helpful discussions, T. Schoberth for experimental assistance and J. Gmeiner for material purification. Financial support from the Bayerische Forschungsstiftung, the Volkswagen Stiftung and the Deutsche Forschungsgemeinschaft is gratefully acknowledged.

[1] A. Bernanose. Electroluminescence of organic compounds Brit. J. Appl. Phys., Suppl. 4:S 54, 1955.

[2] M. Pope, H. Kallmann, and P. Magnante. Electroluminescence in organic crystals. J. Chem. Phys., 38:2042, 1963.

[3] W. Helfrich and W.G. Schneider. Recombination radiation in anthracene crystals. Phys. Rev. Lett., 140:229, 1965 .

[4] D.F. Williams and M. Schadt. A simple organic electroluminescent diode. Proc. IEEE (Lett.), 58:476, 1970.

[5] M. Pope and C.E. Swenberg. Electronic processes in organic crystals. Clarendon Press, Oxford, 1982.

[6] J. Kalinowski. Electroluminescence in organics. J. Phys. D: Appl. Phys., 32:R179, 1999.

[7] N.V. Vityuk and V.V. Mikho. Sov. Phys.Semicond., 6:1479, 1973.

[8] P.S. Vincent, W.A. Barlow, R.A. Hann and G.G. Roberts. Electrical conduction and low voltage blue electroluminescence in vacuum-deposited organic films. Thin Solid Films, 94:476, 1982.

[9] G.G. Roberts, M. McGinnity, P.S. Vincett, and W.A. Barlow. Electroluminescence, photoluminescence and electroabsorption of a lightly substituted anthracene langumuir film. Solid State Communications, 32:683, 1979.

[10] P.H. Partridge. Electroluminescence from polyvinylcarbazole films: 3. Electroluminescent devices. Polymer, 24:748, 1983.

[11] C.W. Tang and S.A. VanSlyke. Organic electroluminescent diodes. Appl. Phys. Lett., 51:913, 1987.

[12] C.W. Tang and S.A. VanSlyke. Electroluminescence of doped organic thin films. J. Appl. Phys., 65:3610, 1989.

[13] C. Adachi, S. Tokito, T. Tsutsui, and S. Saito. Electroluminescence in organic films with threelayer structure. Jpn. J. Appl. Phys., 27:L269, 1988.

[14] C. Adachi, S. Tokito, T. Tsutsui, and S. Saito. Organic electroluminescent device with a threelayer structure. Jpn. J. Appl. Phys., 27:L713, 1988.

[15] C. Adachi, T. Tsutsui, and S. Saito. Electroluminescence mechanism of organic thin film devices. Acta Polymerica, 170:215, 1990.

[16] Pioneer Co. (Japan). In November 1997 Pioneer Co. in Japan commercialized a monochrome 256x64 dot matrix OLED display for automotive applications.

[17] H. Kubota, S. Miyaguchi, S. Ishizuka, T. Wakimoto, J. Funaki, Y. Fukuda, T. Watanabe, H. Ochi, T. Sakamoto, T. Miyake, M. Tsuchida, I. Ohshita, and T. Tohma. Organic LED full color passive-matrix display. Journal of Luminescence, 87-89:56, 2000.

[18] J. Kido. Organic displays. Physics World, 12 (3):27, 1999.

[19] J.H. Burroughes, D.D.C. Bradley, A.R. Brown, R.N. Marks, K. Mackay, R.H. Friend, P.L. Burn, and A.B. Holmes. Light-emitting diodes based on conjugated polymers. Nature, 347:539, 1990.

[20] J.R. Visser. Application of polymer lightemitting materials in light-emitting diodes, backlights and displays. Philips Journal of Research, 51:467, 1998.

[21] R.H. Friend, J. Burroughes, and T. Shimoda. Polymer diodes. Physics World, 12 (6):35, 1999.

[22] S. Karg, M. Meier, and W. Rieß. Light emitting diodes based on poly-p-phenylene-vinylene: I. Charge-carrier injection and transport. J. Appl. Phys., 82:1951, 1997.

[23] M. Meier, S. Karg, and W. Rieß. Light emitting diodes based on poly-p-phenylene-vinylene: II. Impedance spectroscopy. J. Appl. Phys., 82:1961, 1997.

[24] W. Brütting, M. Meier, M. Herold, S. Karg, and M. Schwoerer. Doping in PPV light-emitting devices fabricated on different substrates. Chem. Phys., 227:243, 1998.

[25] J. Scherbel, P.H. Nguyen, G. Paasch, W. Brütting, and M. Schwoerer. Temperature dependent broadband dielectric spectroscopy on poly(p-phenylenevinylene) light-emitting diodes. J. Appl. Phys., 83:5045, 1998.

[26] T.A. Skotheim. Handbook of Conducting Polymers. M. Dekker, New York, 1986.

[27] J. Blochwitz, M. Pfeiffer, T. Fritz, and K. Leo. Low voltage organic light emitting diodes featuring doped phthalocyanine as hole transport material. Appl. Phys. Lett., 73:729, 1998.

[28] A. Nollau, M. Pfeiffer, T. Fritz, and K. Leo. Con- 
trolled n-type doping of a molecular organic semiconductor: Naphtalenetetracarboxylic dianhydride (NTCDA) doped with bis(ethylenedithio)tetrathiafulvalene (BEDT-TTF). J. Appl. Phys., $87: 4340,2000$.

[29] N. Karl. Getting beyond impurity-limited transport in organic photoconductors. In K. Sumino (Ed.), Defect Control in Semiconductors. Elsevier, 1990, p. 1725.

[30] H. Ishii, K. Sugiyama, E. Ito, and K. Seki. Energy level alignment and interfacial electronic structures at organic/metal and organic/organic interfaces. Adv. Mat., 11:605, 1999.

[31] A. Rajagopal, C. I. Wu, and A. Kahn. Energy level offset at organic semiconductor heterojunctions. J. Appl. Phys., 83:2649, 1998.

[32] Y.N. Gartstein, and E.M. Conwell. Fielddependent thermal injection into a disordered molecular insulator. Chem. Phys. Lett., 255:93, 1996.

[33] U. Wolf, V. I. Arkhipov, and H. Bässler. Current injection from a metal to a disordered hopping system. I. Monte carlo simulation. Phys. Rev. B, 59:7507, 1999.

[34] P. M. Borsenberger and D.S. Weiss. Organic Photoreceptors for Imaging Systems. M. Dekker, New York, 1993.

[35] M.A. Lampert and P. Mark. Current Injection in Solids. Academic Press, New York, 1970.

[36] K.C. Kao and W. Hwang. Electrical Transport in Solids. Pergamon Press, Oxford, 1981.

[37] U. Albrecht and H. Bässler. Efficiency of charge recombination in organic light emitting diodes. Chem. Phys., 199:207, 1995.

[38] U. Albrecht and H. Bässler. Langevin-type charge carrier recombination in a disordered hopping system. phys. stat. sol. (b), 191:455, 1995.

[39] M.A. Baldo, S. Lamansky, P.E. Burrows, M.E. Thompson, and S.R. Forrest. Very high-efficiency green organic light-emitting devices based on electrophosphorescence. Appl. Phys. Lett., 75:4, 1999.

[40] T. Tsutsui, M.J. Yang, M. Yahiro, K. Nakamura, T. Watanabe, T. Tsuji, Y. Fukuda, T. Wakimoto, and S. Miyaguchi. High quantum efficiency in organic light-emitting devices with iridiumcomplex as a triplet emissive center. Jpn. J. Appl. Phys., 38, 2000.

[41] S.M. Sze. Physics of Semiconductor Devices. Wiley, New York, 1981.

[42] V.I. Arkhipov, E.V. Emelianova, Y.H. Tak, and H. Bässler. Charge injection into light-emitting diodes: Theory and experiment. J. Appl. Phys., 84:848, 1998.

[43] N.F. Mott and R.W. Gurney. Electronic Processes in Ionic Crystals. Clarendon Press, Oxford, 1940.

[44] M. Pope and C.E. Swenberg. Electronic processes in organic crystals and polymers. Oxford University Press, Oxford, 1999.

[45] R.H. Young. Trap-free space-charge limited current: Analytical solution for an arbitrary mobility law. Phil. Mag. Lett., 70:331, 1994.

[46] P.N. Murgatroyd. Theory of space-charge limited current enhanced by frenkel effect. J. Phys. D: Appl. Phys., 3:151, 1970.

[47] M. Abkowitz, J.S. Facci, and M. Stolka. Timeresolved space-charge limited injection in a trapfree glassy polymer. Chem. Phys., 177:783, 1993.

[48] P.W.M. Blom, M.J.M. de Jong, and C.T.H.F. Liedenbaum. Device physics of polymer lightemitting diodes. Polymers For Advanced Technologies, 9:390, 1998.

[49] H. Bässler. Injection, transport and recombination of charge carriers in organic light-emitting diodes. Polymers For Advanced Technologies, 9:402, 1998.

[50] J.C. Scott, S. Karg, and S.A. Carter. Bipolar charge and current distributions in organic lightemitting diodes. J. Appl. Phys., 82:1454, 1997.

[51] B.K. Crone, P.S. Davids, I.H. Campbell, and D.L. Smith. Device model investigation of single layer organic light emitting diodes. J. Appl. Phys., 84:833, 1998.

[52] J. Shen and J. Yang. Physical mechanisms in double-carrier trap-charge limited transport processes in organic electroluminescent devices: A numerical study. J. Appl. Phys., 83:7706, 1998.

[53] J. Staudigel, M. Stößel, F. Steuber, and J. Simmerer. A quantitative numerical model of multilayer vapor-deposited organic light-emitting diodes. J. Appl. Phys., 86:3895, 1999.

[54] R.G. Kepler, P.M. Beeson, S.J. Jacobs, R.A. Anderson, M.B. Sinclair, V.S. Valencia, and P.A. Cahill. Electron and hole mobility in tris(8hydroxyquinolinolato-n1,o8) aluminium. Appl. Phys. Lett., 66:3618, 1995.

[55] T. Tsutsui, H. Tokuhisa, and M. Era. Charge carrier mobilities in molecular materials for electroluminescent diodes. Proc. SPIE, 3281:230, 1998.

[56] S. Berleb, W. Brütting, and M. Schwoerer. Anomalous current-voltage characteristics in organic light-emitting devices. Synth. Met., 102:1034, 1999.

[57] M.G. Mason, L.S. Hung, C.W. Tang, S.T. Lee, K.W. Wong, and M. Wang. Characterization of treated indium-tin-oxide surfaces used in electroluminescent devices. J. Appl. Phys., 86:1688, 1999.

[58] K. Sugiyama and H. Ishii and Y. Ouchi and K. Seki. Dependence of indium-tin-oxide work function on surface cleaning method as studied by ultraviolet and x-ray photoemission spectroscopies. J. Appl. Phys., 87:295, 2000.

[59] F. Nüesch, E.W. Forsythe, Q.T. Le, Y. Gao, and L.J. Rothberg. Importance of indium tin oxide surface acido basicity for charge injectioninto organic materials based light emitting diodes. $J$. Appl. Phys., 87:7973, 2000.

[60] T. Beierlein, W. Brütting, H. Riel, E.I. Haskal, P. Müller, and W. Rieß. Kelvin probe investigations of metal work functions and correlation to device performance of organic light-emitting devices. Synth. Metals, 111-112:295, 2000.

[61] T. Virgili, D.G. Lidzey, and D.D.C. Bradley. Efficient energy transfer from blue to red in tetraphenylporphyrin-doped $\operatorname{poly}(9,9-$ dioctylfluorene) light-emitting diodes. Adv. Mat., 
12:58, 2000 .

[62] J. de Mello, F. Wittmann, and R.H. Friend. An improved experimental determination of external photoluminescence quantum efficiency. $A d v$. Mater., 9:230, 1997.

[63] C. Giebeler, S.A. Whitelegg, D.G. Lidzey, P.A. Lane, and D.D.C. Bradley. Device degradation of polymer light emitting diodes studied by electroabsorption measurements. Appl. Phys. Lett., 75:2144, 1999.

[64] A.J. Campbell, D.D.C. Bradley, J. Laubender, and M. Sokolowski. Thermally activated injection limited conduction in single layer N,N'-diphenyl-N,N'-bis(3-methylphenyl)-11'-biphenyl-4,4'-diamine light emitting diodes. $J$. Appl. Phys., 86:5004, 1999.

[65] P.H. Nguyen, S. Scheinert, S. Berleb, W. Brütting, and G. Paasch. The influence of deep traps on transient current-voltage characteristics of organic light-emitting diodes. Phys. Rev. B, submitted, 2000.

[66] A.J. Campbell and D.D.C. Bradley. Transient studies of deep traps in electroluminescent polymers. Proc. SPIE, 3797:326, 1999.

[67] L.S. Roman, M. Berggren, and O. Inganäs. Polymer diodes with high rectification. Appl. Phys. Lett., 75:3557, 1999.

[68] T. Kugler and A. Johansson and I. Dalsegg and U. Gelius and W.R. Salaneck. Electronic and chemical structure of conjugated polymer surfaces and interfaces: Applications in polymerbased light-emitting devices. Synth. Met., 91:143, 1997.

[69] Y. Park, V. Choong, Y.Gao, B.R. Hsieh, and C.W. Tang. Work function of ITO transparent conductor measured by photoelectron spectroscopy. Appl. Phys. Lett., 68:2699, 1996.

[70] T. Osada, T. Kugler, P. Bröms, and W.R. Salaneck. Polymer-based light-emitting devices: Investigations on the role of the indium-tin oxide (ITO) electrode. Synth. Met., 96:77, 1998.

[71] G.G. Malliaras, J.R. Salem, P.J. Brock, and J.C. Scott. Photovoltaic measurement of the built-in potential in organic light emitting diodes and photodiodes. J. Appl. Phys., 84:1583, 1998.

[72] L.-B. Lin, R.H. Young, M.G. Mason, and P.M. Borsenberger. Transient photocurrents across organic-organic interfaces. Appl. Phys. Lett., $72: 864,1998$.

[73] T. Tsutsui. Molecular design for organic light emitting diodes. Speaker's notes, Proc. ICEL-2 (Sheffield), (unpublished), 1999.

[74] B.J. Chen, W.Y. Lai, Z.Q. Gao, C.S. Lee, S.T. Lee, and W.A. Gambling. Electron drift mobility and electroluminescencet efficiency of tris $(8-$ hydroxyquinolinolato) aluminium. Appl. Phys. Lett., 75:4010, 1999.

[75] J. Staudigel, M. Stössel, F. Steuber, and J. Simmerer. Comparison of mobility and hole current activation energy in the space charge trap-limited regime in a starburst amine. Appl. Phys. Lett., 75:217, 1999.

[76] U. Wolf, S. Barth, and H. Bässler. Electrode versus space-charge-limited conduction in organic light-emitting diodes. Appl. Phys. Lett., 75:2035, 1999.

[77] N.S. Sariciftci (Ed.). Primary Photoexcitations in Conjugated Polymers: Molecular Exciton versus Semiconductor Band Model. World Scientific, Singapore, 1997.

[78] A. Curioni, M. Boero, and W. Andreoni. Alq ${ }_{3}$ : $\mathrm{Ab}$ initio calculations of its structural and electronic properties in neutral and charged states. Chem. Phys. Lett., 294:263, 1998.

[79] M. Brinkmann, G. Gadret, M. Muccini, C. Taliani, N. Masciocchi, and A. Sironi. Correlation between molecular packing and optical properties in different crystalline polymorphs and amorphous thin films of mer-tris(8hydroxyquinoline)aluminium(III). J. Am. Chem. Soc., 122:5147, 2000.

[80] S. Barth, U. Wolf, H. Bässler, P. Müller, H. Riel, H. Vestweber, P.F. Seidler, and W. Rieß. Current injection from a metal to a disordered hopping system. III. Comparison between experiment and monte carlo simulation. Phys. Rev. B, 60:8791, 1999.

[81] P.E. Burrows, Z. Shen, V. Bulovic, D.M. McCarty, S.R. Forrest, J.A. Cronin, and M.E. Thompson. Relationship between electroluminescence and current transport in organic heterojunction LEDs. J. Appl. Phys., 79:7991, 1996.

[82] M. Stößel, J. Staudigel, F. Steuber, J. Blässing, J. Simmerer, and A. Winnacker. Space-chargelimited electron currents in 8-hydroxyquinoline aluminium. Appl. Phys. Lett., 76:115, 2000.

[83] S. Berleb, A.G. Mückl, W. Brütting, and M. Schwoerer. Temperature dependent device characteristics of organic light emitting devices. Synth. Metals, 111-112:341, 2000.

[84] S. Naka, H. Okada, H. Onnagawa, J. Kido, and T. Tsutsui. Time-of-flight measurement of hole monility in aluminium (III) complexes. Jpn. J. Appl. Phys., 38:L1252, 1999.

[85] A. Ioannidis, E. Forsythe, Y. Gao, M.W. Wu, and E.M. Conwell. Current-voltage characteristic of organic light emitting diodes. Appl. Phys. Lett., 72:3038, 1998.

[86] V.I. Arkhipov, U. Wolf, and H. Bässler. Current injection from a metal to a disordered hopping system. II. Comparison between analytic theory and simulation. Phys. Rev. B, 59:7514, 1999.

[87] I.H. Campbell and D.L. Smith. Schottky energy barriers and charge injection in metal/Alq/metal structures. Appl. Phys. Lett., 74:561, 1999.

[88] A.J. Campbell, M.S. Weaver, D.G. Lidzey, and D.D.C. Bradley. Bulk limited conduction in electroluminescent polymer devices. J. Appl. Phys., 84:6737, 1998.

[89] W.D. Gill. Drift mobilities in amorphous charge-transfer complexes of trinitrofluorenone and poly-n-vinylcarbazole. J. Appl. Phys., 55(12):5033, 1972.

[90] H. Böttger and V.V. Bryksin. Hopping Conduction in Solids. VCH, Weinheim (Germany), 1985.

[91] W. Brütting, S. Berleb, and A.G. Mückl. Spacecharge limited conduction with a field and temperature dependent mobility in Alq light- 
emitting devices. Proc. E-MRS 2000, Synth. Met., in press, 2001.

[92] S. Karg, J. Steiger, and H. von Seggern. Determination of trap energies in $\mathrm{Alq}_{3}$ and TPD. Synth. Met., 111-112:277, 2000.

[93] E.W. Forsythe, D.C. Morton, C.W. Tang, and Y. Gao. Trap states of tris-8-(hydroxyquinoline) aluminum and naphthyl-substituted benzidine derivative using thermally stimulated luminescence. Appl. Phys. Lett., 73:1457, 1998.

[94] E.A. Silinsh. Organic Molecular Crystals. Springer, Berlin, 1980.

[95] C. Schmitz, P. Pösch, M. Thelakkat, and H.-W. Schmidt. Efficient screening of electron transport material in multi-layer organic light emitting diodes by combinatorial methods. Phys. Chem. Chem. Phys., 1:1777, 1999.

[96] T. Tsutsui. Progress in electroluminescent devices using molecular thin films. MRS Bulletin, 22(6):39, 1997.

[97] N.C. Greenham, R.H. Friend, and D.D.C. Bradley. Angular dependance of the emission from a conjugated LED: Implications for efficiency calculations. Adv. Mat., 6:491, 1994.

[98] M. Stolka, J.F. Yanus, and D.M. Pai. Hole transport in solid solutions of a diamine in polycarbonate. J. Phys. Chem., 88:4707, 1984.

[99] B. Chen and S. Liu. Measurement of electron/hole moblity in organic/polymeric thin films using modified time-of-flight apparatus. Synth. Met., 91:169, 1997.

[100] L.-B. Lin, S.A. Jenekhe, R.H. Young, and P.M. Borsenberger. Hole injection and transport in tris-(8-hydroxyquinolinato) aluminium. Appl. Phys. Lett., 70:2052, 1997.

[101] C. Hosokawa, H. Tokailin, H. Higashi, and T. Kusumoto. Transient behavior of organic thin film EL. Appl. Phys. Lett., 60:1220, 1992.

[102] H. Vestweber, J. Oberski, A. Greiner, W. Heitz, R.F. Mahrt, and H. Bässler. Electroluminescence from phenylenevinylene-based polymer blends. Advanced Materials for Optics and Electronics, 2:197, 1993.

[103] S. Karg, V. Dyakonov, M. Meier, W. Rieß and G. Paasch. Transient electroluminescence in poly $(\mathrm{p}-$ phenylene vinylene) light-emitting diodes. Synth. Met., 67:165, 1994.

[104] P. Ranke, I. Bleyl, J. Simmerer, D. Haarer, A Bacher, and H.-W. Schmidt. Electroluminescence and electron transport in a perylene dye. Appl. Phys. Lett., 71:1332, 1997.

[105] D.J. Pinner, R.H. Friend, and N. Tessler. Transient electroluminescence of polymer light emitting diodes using electrical pulses. J. Appl. Phys., $86: 5116,1999$

[106] H. Kurczewska and H. Bässler. Energy transfer across an anthracene-gold interface. J. Lumin., 15:261, 1977.

[107] J. Grüner, M. Remmers, and D. Neher. Direct determination of the emission zone in a polymer light-emitting diode. Adv. Mater., 9:964, 1997.

[108] H. Becker, S.E. Burns, and R.H. Friend. Effect of metal films on the photoluminescence and electroluminescence of conjugated polymers. Phys.
Rev. B, 56:1893, 1997.

[109] V.R. Nikitenko, Y.-H. Tak, and H. Bässler. Rise time of electroluminescence from bilayer light emitting diodes. J. Appl. Phys., 84:2334, 1998.

[110] W. Brütting, H. Riel, T. Beierlein, and W. Rieß. Influence of trapped and interfacial charges in organic multi-layer light-emitting devices. J. Appl. Phys., 15 Jan. 2001.

[111] H. Nakamura, C. Hosokawa, and T. Kusumoto. Transient behaviour of organic electroluminescent cells. In R.H. Mauch and H.-E. Gumlich (Eds.), Inorganic and Organic Electroluminescence. Wissenschaft \& Technik Verlag, Berlin, 1996.

[112] H. Bässler. Charge transport in disordered organic photoconductors. phys. stat. sol. (b), 175:15, 1993.

[113] D.H. Dunlap, P.E. Parris, and V.M. Kenkre. Charge-dipole model for the universal field dependence of mobilities in molecularly doped polymers. Phys. Rev. Lett., 77:542, 1996.

[114] S.V. Novikov, D.H. Dunlap, V.M. Kenkre, P.E. Parris, and A.V. Vannikov. Essential role of correlations in governing charge transport in disordered organic materials. Phys. Rev. Lett., 81:4472, 1998.

[115] D.H. Dunlap, V.M. Kenkre, and P.E. Parris. What is behind the $\sqrt{E}$ ?. J. Imag. Sc. Techn., 43:437, 1999 .

[116] D. Hertel and H. Bässler and U. Scherf. H.H. Hörhold. Charge carrier transport in conjugated polymers. J. Chem. Phys., 110:9214, 1999.

[117] P.W.M. Blom, M.J.M. de Jong, and M.G. van Munster. Electric-field and temperature dependence of the hole mobility in poly (p-phenylene vinylene). Phys. Rev. B, 55:656, 1997.

[118] P.W.M. Blom and M.C.J.M. Vissenberg. Dispersive hole transport in poly (p-phenylene vinylene). Phys. Rev. Lett., 80:3819, 1998.

[119] Y. Shirota and S. Nomura and H. Kageyama. Charge transport in amorphous molecular materials. Proc. SPIE, 3476:132, 1999.

[120] J. Kanicki. Polymer semiconductor contacts and photovoltaic applications. In T.A. Skotheim (Ed.), Handbook of Conducting Polymers Vol.1. M. Dekker, New York, 1986.

[121] D.M. Taylor and H.L. Gomes. Electrical characterization of the rectifying contact between aluminium and electrodeposited poly(3methylthiophene). J. Phys. D: Appl. Phys., 28:2554, 1995 .

[122] A.J. Campbell, D.D.C. Bradley, E. Werner, and W. Brütting. Transient capacitance measurements of the transport and trap states distributions in a conjugated polymer. Organic Electron$i c s$, in press, 2000.

[123] M. Matsumura, A. Ito, and Y. Miyamae. Accumulation of positive charges in organic lightemitting diodes with a double-layer structure. Appl. Phys. Lett., 75:1042, 1999.

[124] F. Rohlfing, T. Yamada, and T. Tsutsui. Electroabsorption spectroscopy on tris(8-hydroxyquinoline) aluminum-based light emitting diodes. J. Appl. Phys., 86:4978, 1999. 
[125] A.G. Mückl, S. Berleb, W. Brütting, and M. Schwoerer. Transient electroluminescence measurements on organic hetero layer light emitting diodes. Synth. Metals, 111-112:91, 2000.

[126] S. Scheinert, G. Paasch, S. Berleb, and W. Brütting. unpublished, 2000.

\section{FIGURES}

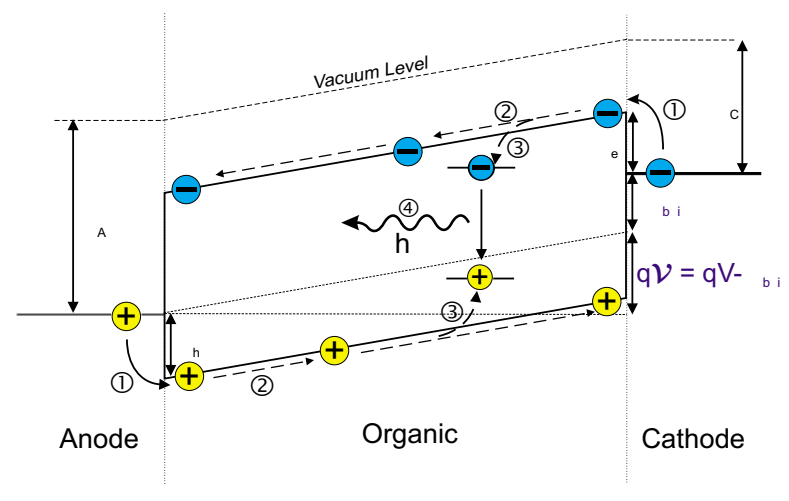

FIG. 1. Basic steps of electroluminescence: (1) charge carrier injection, (2) charge carrier transport, (3) exciton formation, (4) radiative exciton decay. Polaronic effects and a distribution of transport states due to disorder are neglected. $\left(\Phi_{A}\right.$ : anode work function, $\Phi_{C}$ : cathode work function, $\Phi_{h}$ : hole injection barrier, $\Phi_{e}$ : electron injection barrier, $\Phi_{b i}$ : built-in potential, $V$ : applied voltage, $\mathcal{V}$ : effective voltage across the organic layer, $q$ : elementary charge).

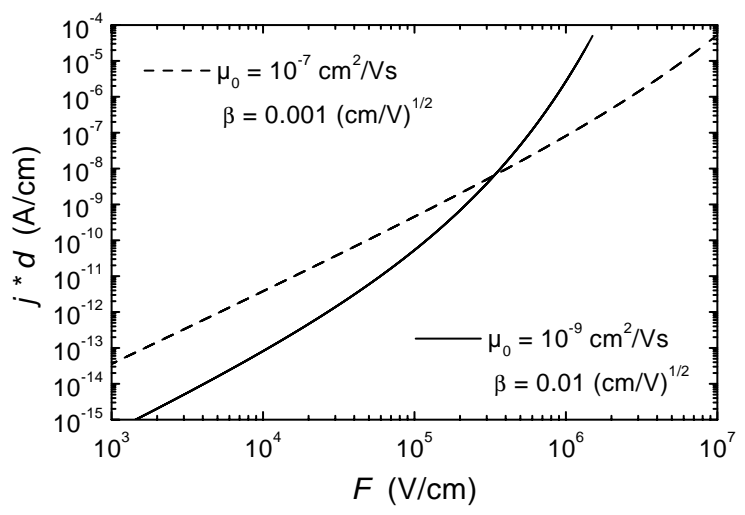

FIG. 2. Simulated current-voltage characteristics for trap-free SCLC with a field-dependent mobility $\mu(F)=\mu_{0} \exp (\beta \sqrt{F})$ for two typical parameter sets for the materials under investigation. In the given representation there is no difference between curves for layer thicknesses between 50 and $500 \mathrm{~nm}$ which proves that for trap-free SCLC (including a field-dependent mobility) the current at constant electric field scales with $d^{-1}$.

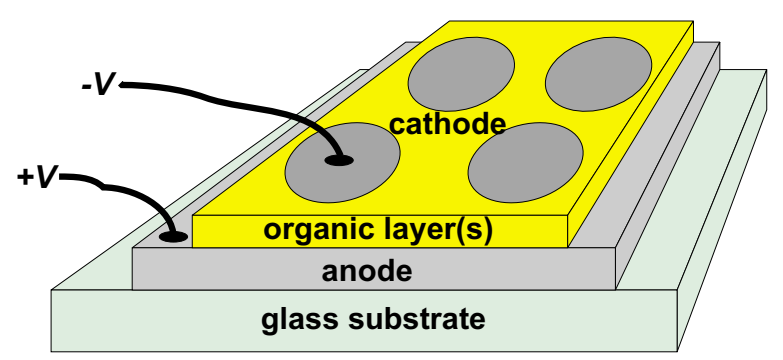

FIG. 3. Typical device structure used in this work with 4 active pixels each about $10 \mathrm{~mm}^{2}$ large. ITO or Al were used as anode and $\mathrm{Ca}$ or $\mathrm{Al}$ as cathode contacts. The typical overall organic layer thickness was around 100 to $400 \mathrm{~nm}$. The lateral dimensions of the substrate were $20 \mathrm{~mm} \times 20 \mathrm{~mm}$.
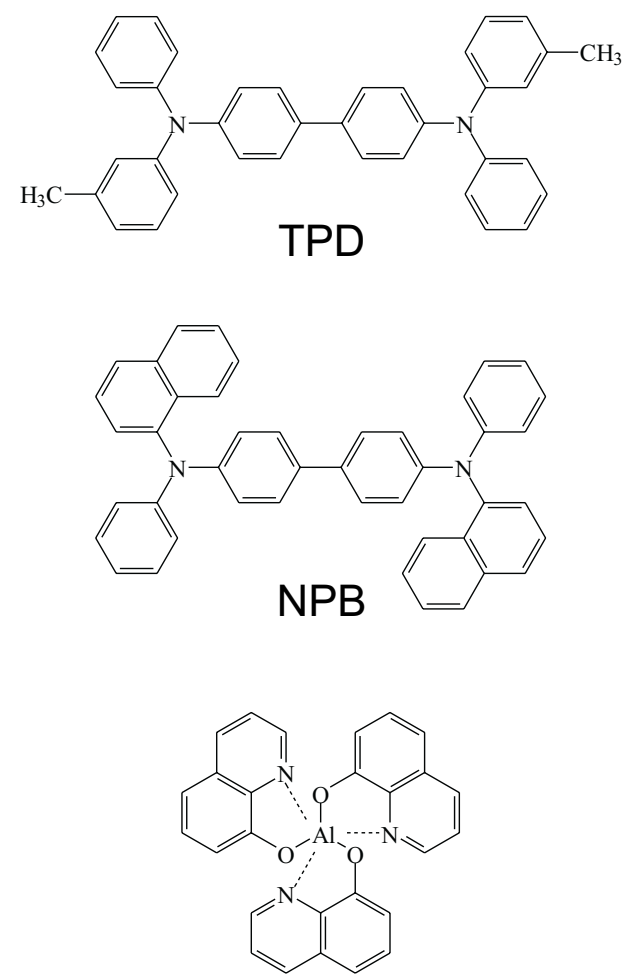

Alq

FIG. 4. Chemical structures of the materials used in this work. TPD: N,N'-diphenyl-N,N'-bis (3-methylphenyl)-1,1'-biphenyl-4,4'-diamine, NPB: N,N'-diphenyl-N,N'-bis(1-naphthyl)-1,1'-biphenyl-4,4'-diamine, Alq: tris(8-hydroxyquinolato)aluminium. 


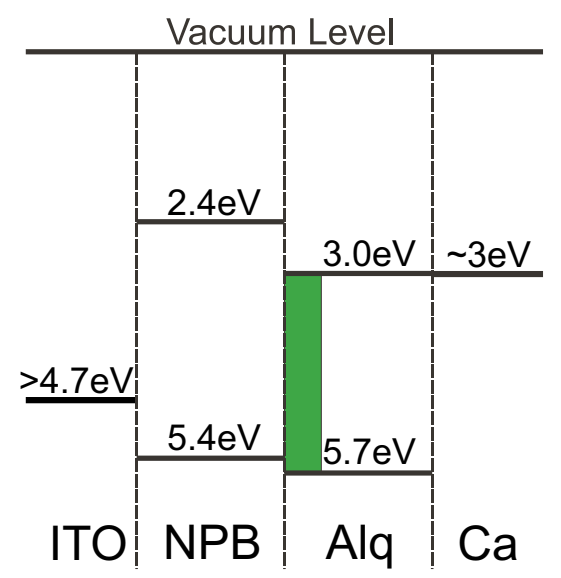

FIG. 5. Schematic energy level diagram of an NPB/Alq hetero-layer device. The shaded area indicates the position of the recombination zone in Alq.

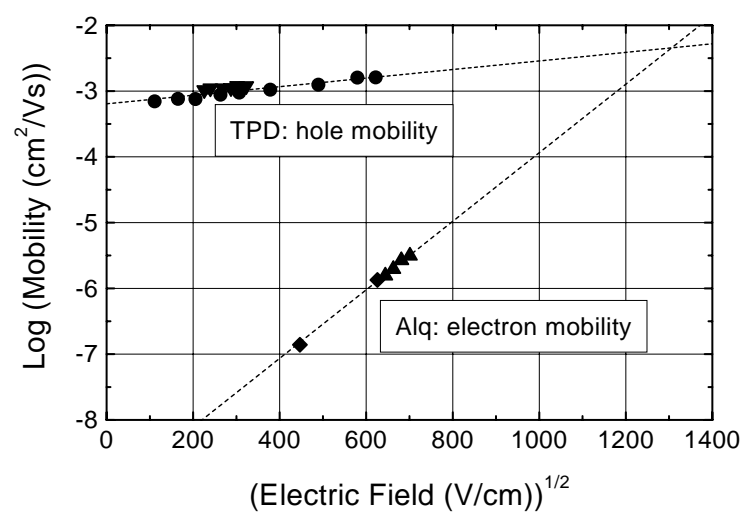

FIG. 6. Charge carrier mobility in TPD and Alq obtained by the time-of-flight technique by different authors (from [54,55]).
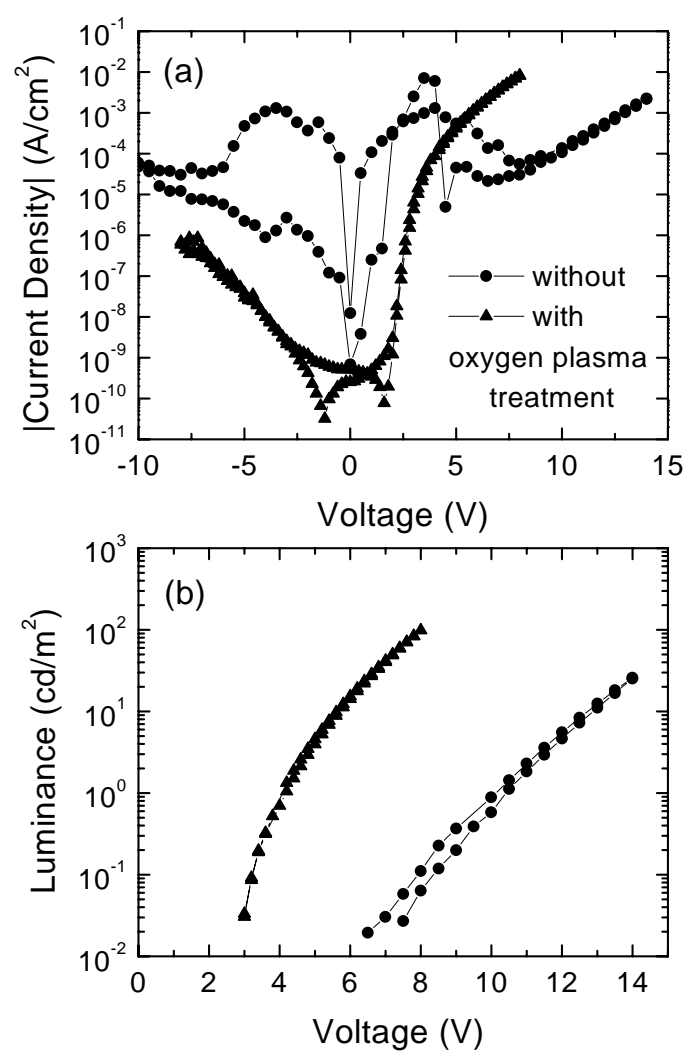

FIG. 7 (a) Current-voltage and (b) luminance-voltage characteristics of one of our early ITO/TPD/Alq/Ca hetero-layer devices without and with oxygen plasma treated ITO anodes.

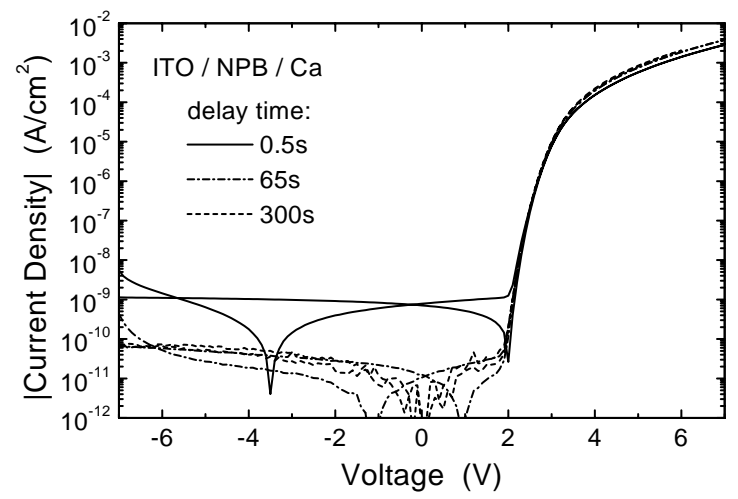

FIG. 8. Current-voltage characteristics of an ITO/NPB/Ca single-layer device with a 430nm thick NPB layer measured in voltage steps of $0.1 \mathrm{~V}$ with various delay times between individual data points. For each delay time a curve measured for increasing and decreasing voltage as indicated by the arrows is shown. 


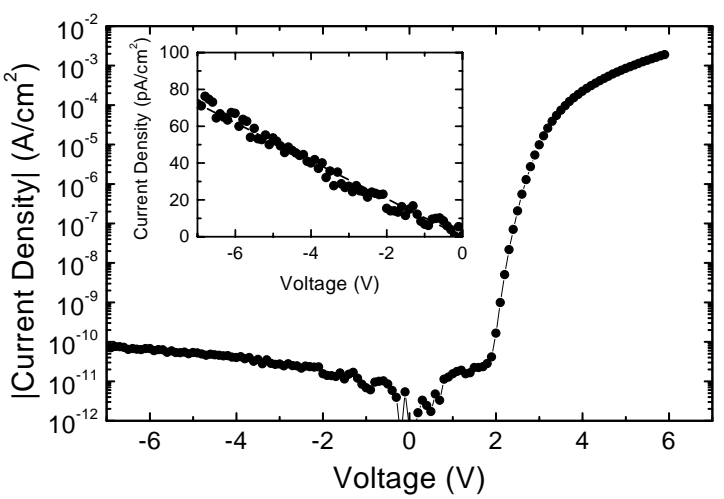

FIG. 9. Equilibrium current-voltage characteristics of the same ITO/NPB/Ca single-layer device as shown in Fig. 8, measured with a delay time of 300 s between individual data points. The inset shows the reverse current on a linear scale.
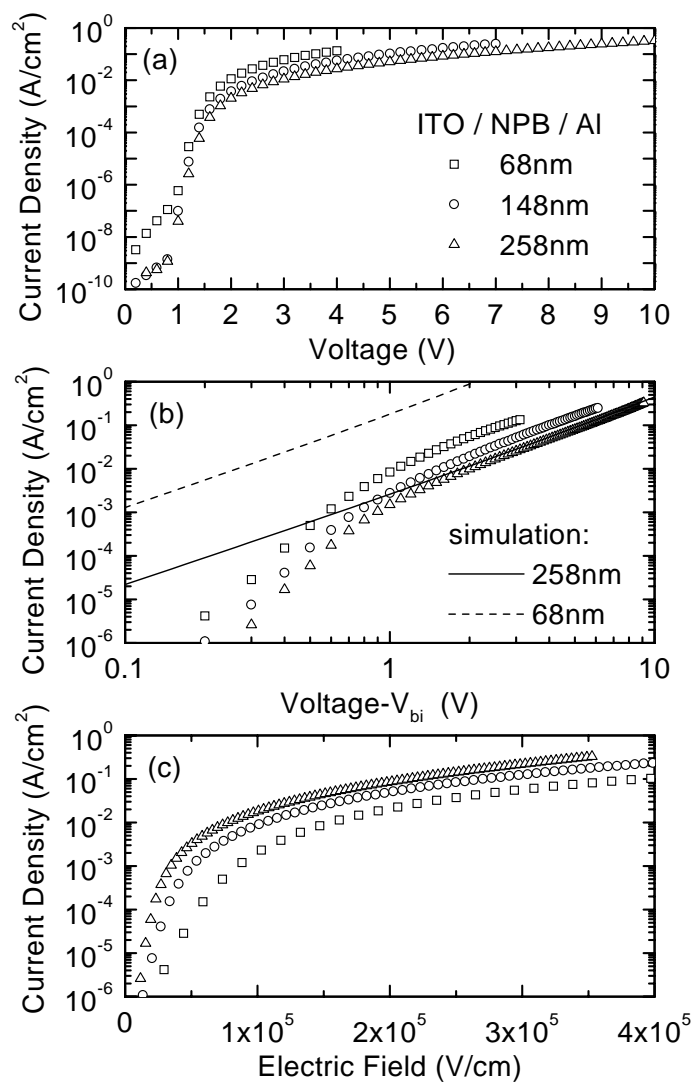

FIG. 10 Current-voltage characteristics of ITO/NPB/Al hole-only devices with different NPB layer thickness: (a) semi-logarithmic representation, (b) double-logarithmic representation with simulated currents for SCLC with a field-dependent mobility $\mu(F)=\mu_{0} \exp (\beta \sqrt{F})$ with $\mu_{0}=10^{-4} \mathrm{~cm}^{2} / \mathrm{Vs}$ and $\beta=0.0015(\mathrm{~cm} / \mathrm{V})^{1 / 2}$, (c) current vs. electric field.
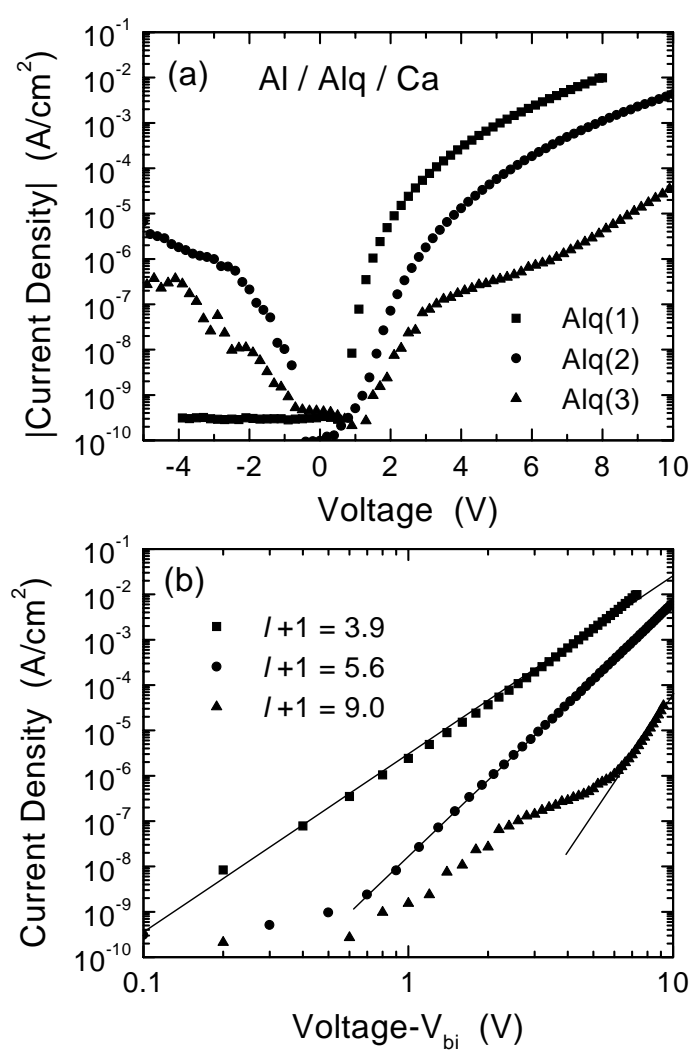

FIG. 11. Current-voltage characteristics of $\mathrm{Al} / \mathrm{Alq} / \mathrm{Ca}$ electron-only devices made with different Alq: (a) semi-logarithmic representation, (b) double-logarithmic representation with fits to power laws $j \propto \mathcal{V}^{l+1}$. All devices had about the same Alq thickness of $90 \mathrm{~nm}$. 


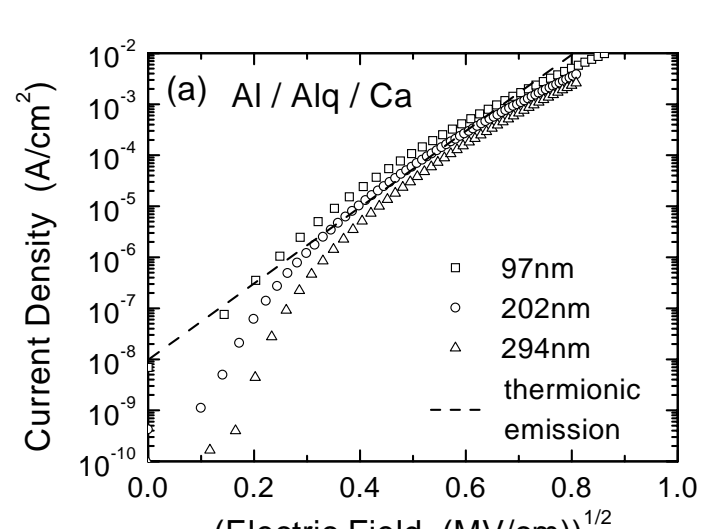

(Electric Field $(\mathrm{MV} / \mathrm{cm}))^{1 / 2}$

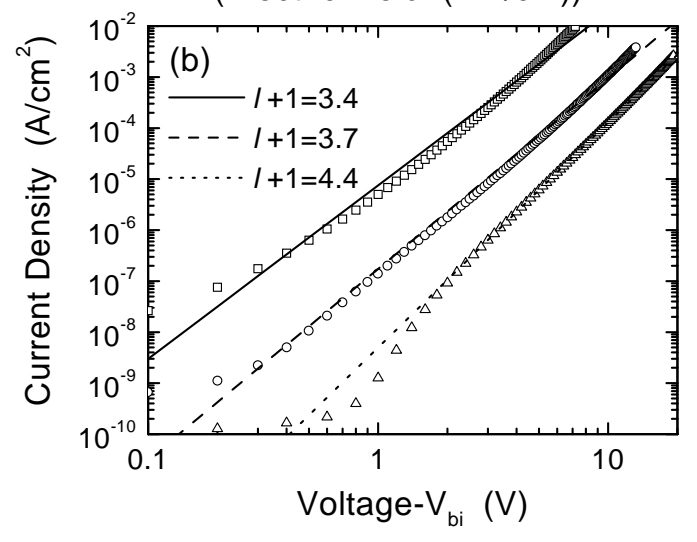

FIG. 12. Current-voltage characteristics of $\mathrm{Al} / \mathrm{Alq} / \mathrm{Ca}$ electron-only devices with different Alq layer thickness: (a) Richardson-Schottky plot, (b) double-logarithmic representation with fits to power laws $j \propto \mathcal{V}^{l+1}$.

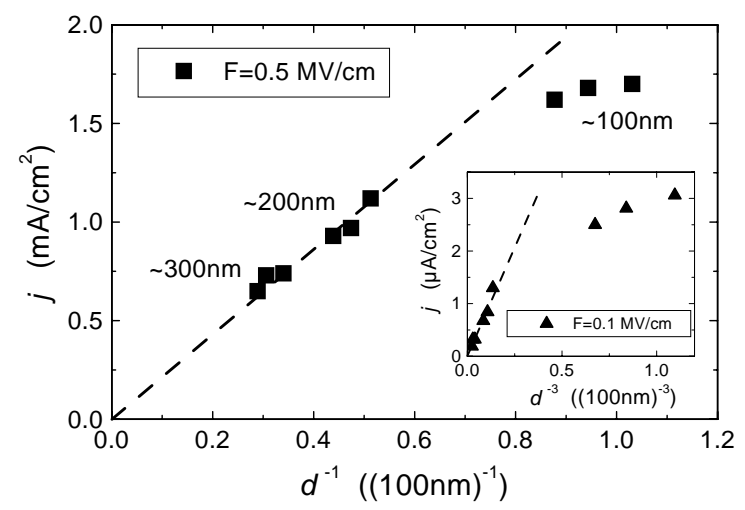

FIG. 13. Thickness dependence of the current $(j$ vs. $\left.d^{-1}\right)$ at a constant electric field of $0.5 \mathrm{MV} / \mathrm{cm}$ for $\mathrm{Al} / \mathrm{Alq} / \mathrm{Ca}$ electron-only devices. The inset shows a plot of $j$ vs. $d^{-3}$ at an electric field of $0.1 \mathrm{MV} / \mathrm{cm}$.

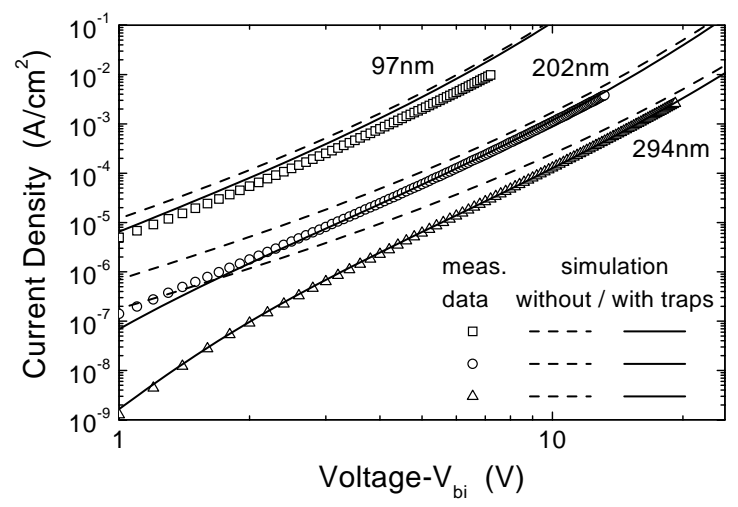

FIG

14.

Current-voltage characteristics of $\mathrm{Al} / \mathrm{Alq} / \mathrm{Ca}$ electron-only devices with different Alq layer thickness in double-logarithmic representation. The dashed lines are simulated currents for trap-free SCLC with a field-dependent mobility $\mu(F)=\mu_{0} \exp (\beta \sqrt{F})$ with $\mu_{0}=2 \times 10^{-9} \mathrm{~cm}^{2} / \mathrm{Vs}$ and $\beta=0.008(\mathrm{~cm} / \mathrm{V})^{1 / 2}$. The solid lines are simulations of SCLC with the same mobility parameters but including an exponential trap distribution with $N_{t}=1.3 \times 10^{17} \mathrm{~cm}^{-3}$ and $E_{t}=0.14 \mathrm{eV}$.
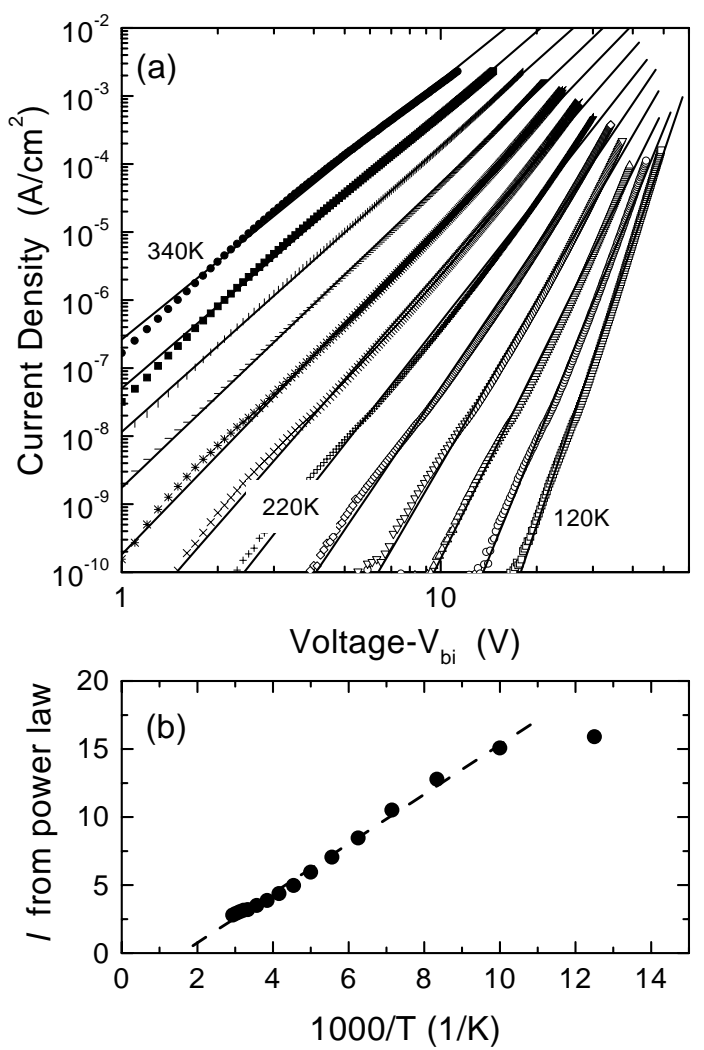

FIG. 15. (a) Temperature dependence of the current-voltage characteristics of an $\mathrm{Al} / \mathrm{Alq} / \mathrm{Ca}$ electron-only device with an Alq layer thickness of 300nm in double-logarithmic representation with fits to power laws $j \propto \mathcal{V}^{l+1}$. (b) Temperature dependence of the power law exponents $l$ obtained from (a). 

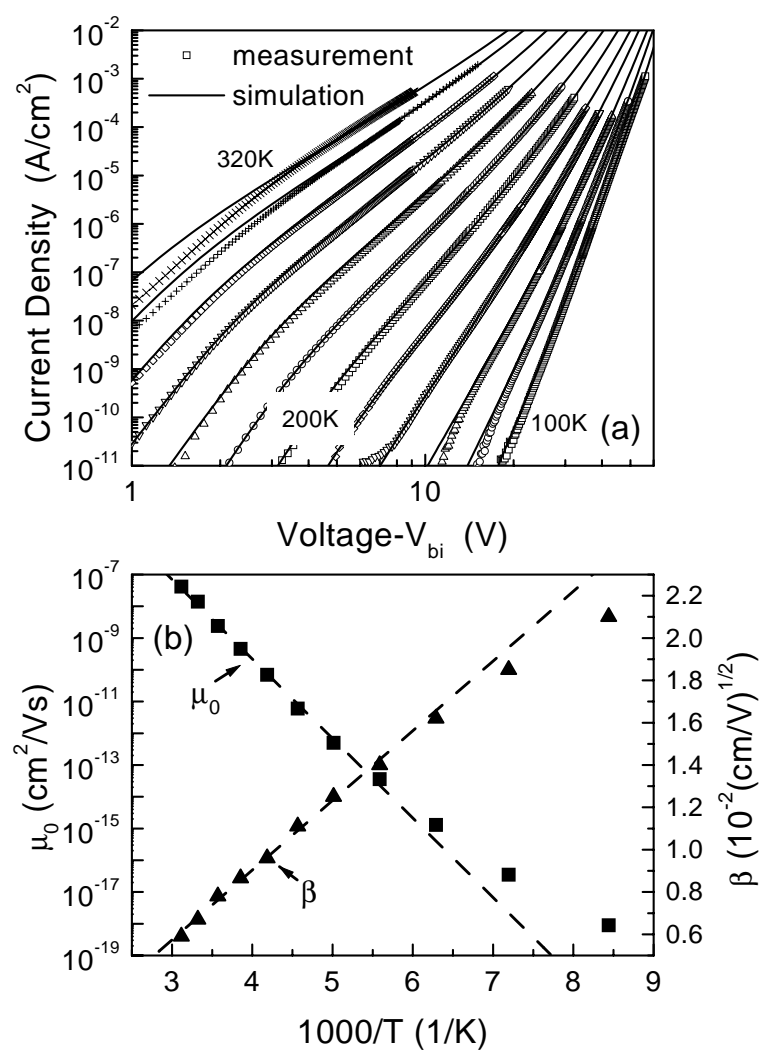

FIG. 16. (a) Temperature dependence of the current-voltage characteristics of an $\mathrm{Al} / \mathrm{Alq} / \mathrm{Ca}$ electron-only device with an Alq layer thickness of 300nm in double-logarithmic representation. The lines are simulated currents for SCLC with a field-dependent mobility $\mu(F)=\mu_{0} \exp (\beta \sqrt{F})$ and an exponential trap distribution with $N_{t}=1.3 \times 10^{17} \mathrm{~cm}^{-3}$ and $E_{t}=0.14 \mathrm{eV}$. (b) Temperature dependence of the mobility parameters $\mu_{0}$ and $\beta$ obtained from (a) together with fit curves according to the Poole-Frenkel model.
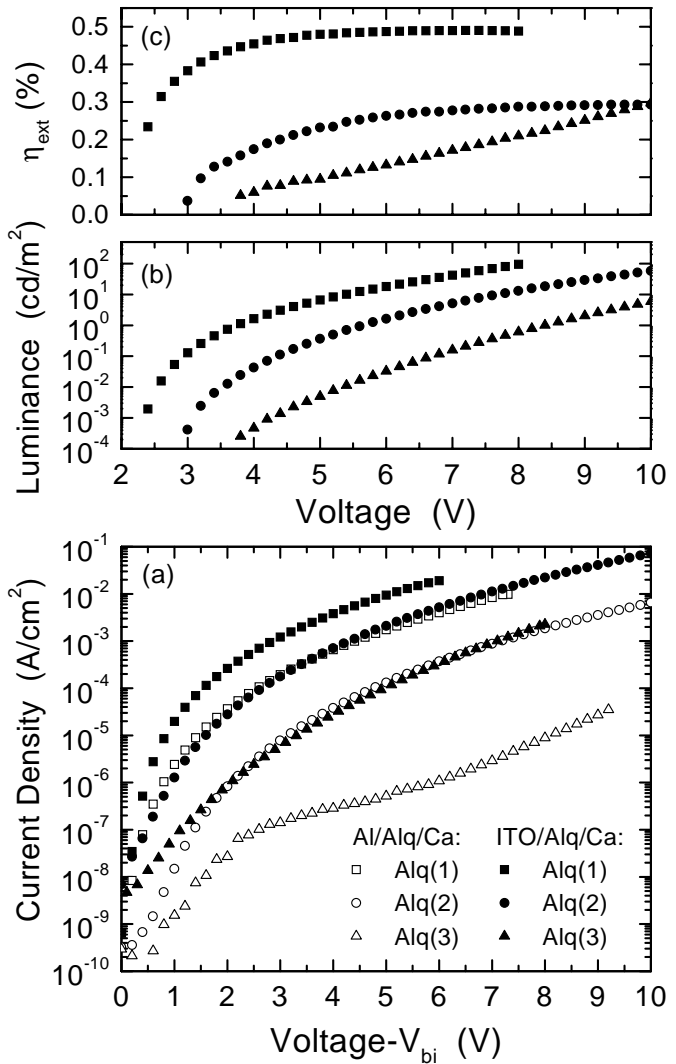

FIG. 17. (a) Current-voltage characteristics, (b) luminance-voltage characteristics and (c) external EL quantum efficiency as a function of voltage of ITO/Alq/Ca devices with different Alq. The currents for the corresponding $\mathrm{Al} / \mathrm{Alq} / \mathrm{Ca}$ devices from Fig. 11 are shown again. All devices had about the same Alq thickness of $90 \mathrm{~nm}$. 

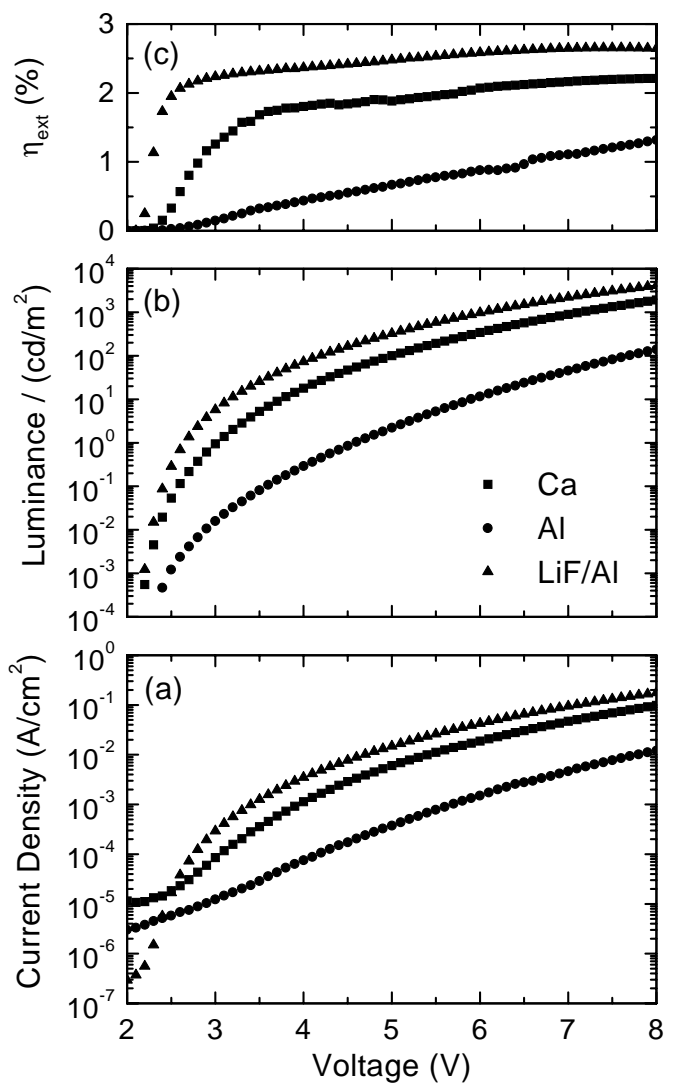

FIG. 18. (a) Current-voltage characteristics, (b) luminance-voltage characteristics and (c) external EL quantum efficiency as a function of voltage of ITO/NPB $(30 \mathrm{~nm}) / \mathrm{Alq}(60 \mathrm{~nm})$ hetero-layer devices with different cathodes.

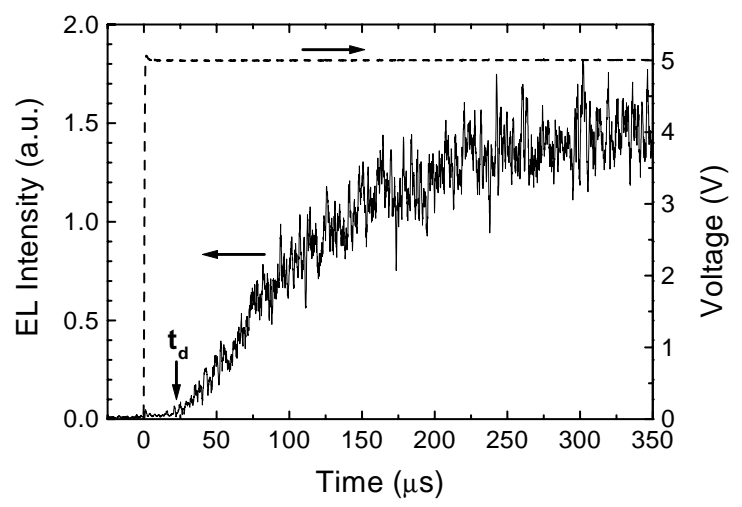

FIG. 19. Delayed electroluminescence from an ITO/TPD/Alq/Ca device with $60 \mathrm{~nm}$ TPD and $60 \mathrm{~nm}$ Alq after application of a single voltage pulse (dashed line) with an amplitude of $5 \mathrm{~V}$ at $240 \mathrm{~K}$. The delay time $t_{d}$ is approximately $20 \mu \mathrm{s}$.

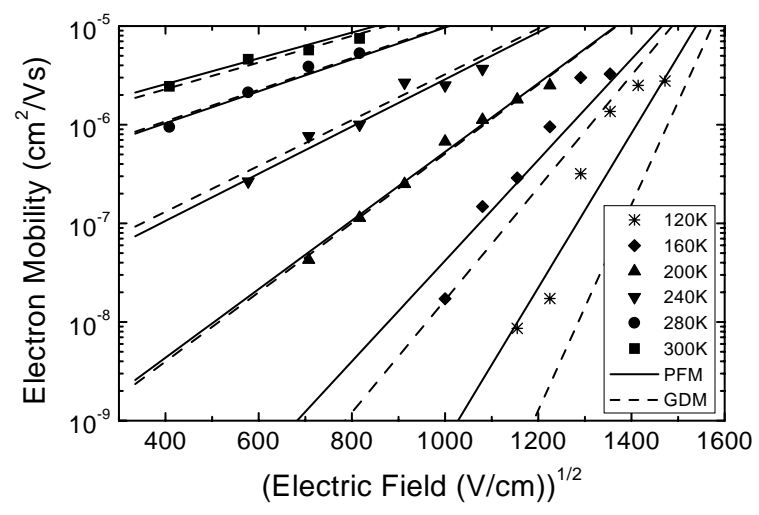

FIG. 20. Temperature and field dependence of the electron drift mobility in Alq as obtained from transient electroluminescence. Also included are the results of the analysis with the Poole-Frenkel model (PFM) and the Gaussian disorder model (GDM).

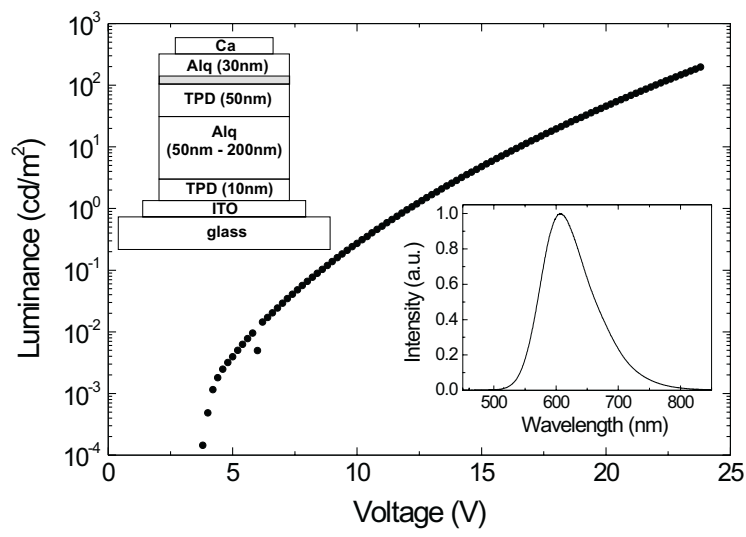

FIG. 21. Electroluminescence characteristics of the double hetero structure device with a $50 \mathrm{~nm}$ Alq layer sandwiched between two TPD layers as shown in the inset. The upper Alq layer is partially doped with $2 \%$ of the red laser dye DCM2. The doped region is indicated by the hatching. The inset shows the emission spectrum of this structure.

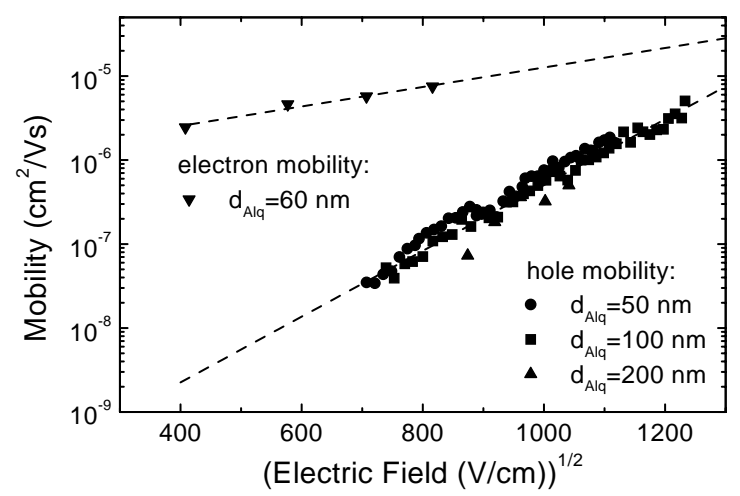

FIG. 22. Field dependence of charge carrier mobilities in Alq obtained by transient EL at $300 \mathrm{~K}$. 


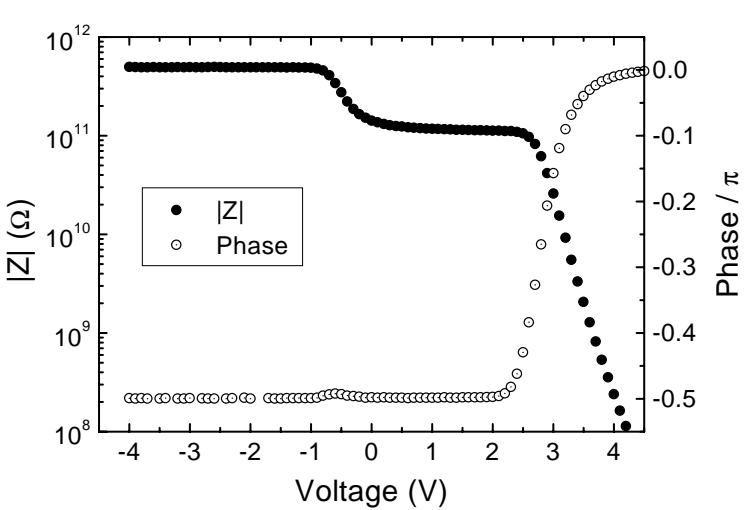

FIG. 23. Modulus $|\hat{Z}|$ and phase $\varphi$ of the complex impedance of an ITO/NPB/Alq/Ca device with $60 \mathrm{~nm}$ NPB and $60 \mathrm{~nm}$ Alq as a function of the applied bias, measured at a frequency of $100 \mathrm{~Hz}$.

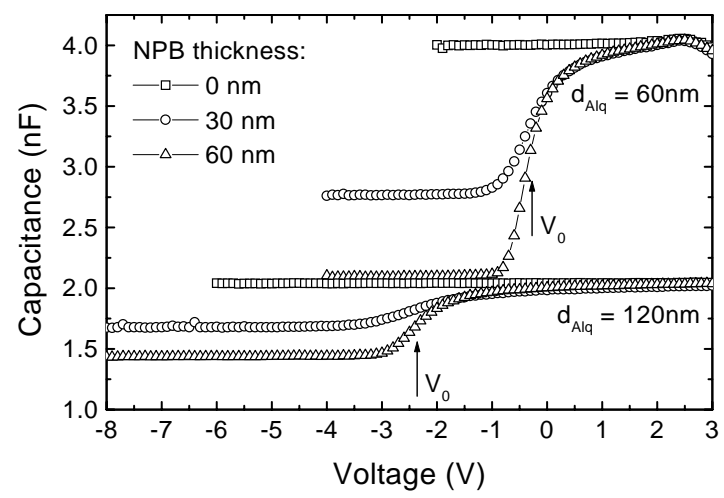

FIG. 24. Bias-dependent device capacitance of a series of ITO/NPB/Alq/Ca devices with different NPB and Alq layer thickness.

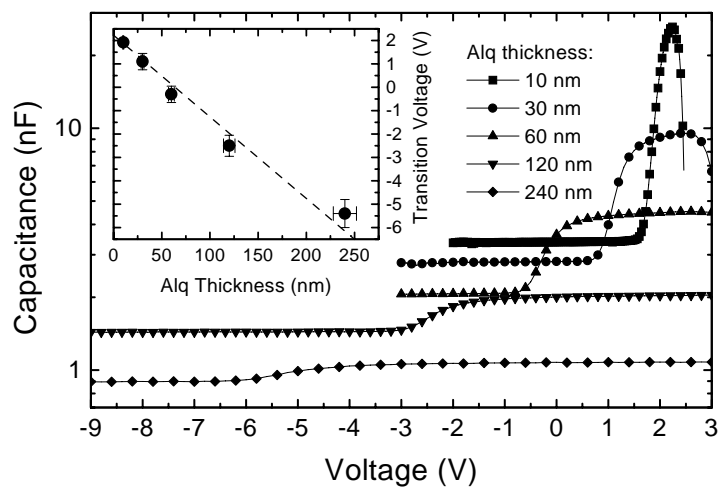

FIG. 25. Bias-dependent device capacitance of a series of ITO/NPB/Alq/Ca devices with a $60 \mathrm{~nm}$ thick NPB layer and different Alq layer thickness. The inset shows the dependence of the transition voltage on the Alq layer thickness.

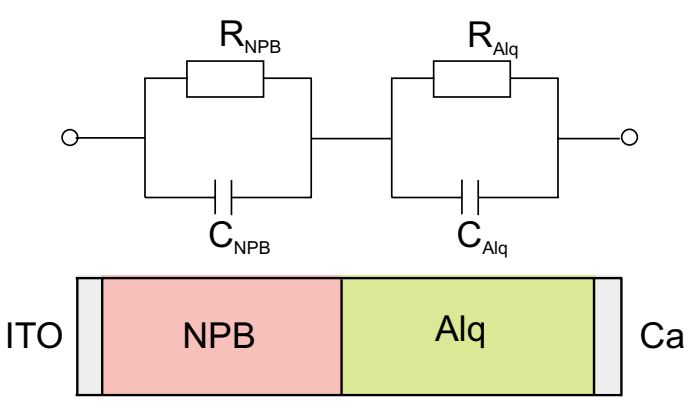

FIG. 26. Equivalent circuit of an ITO/NPB/Alq/Ca hetero-layer device.

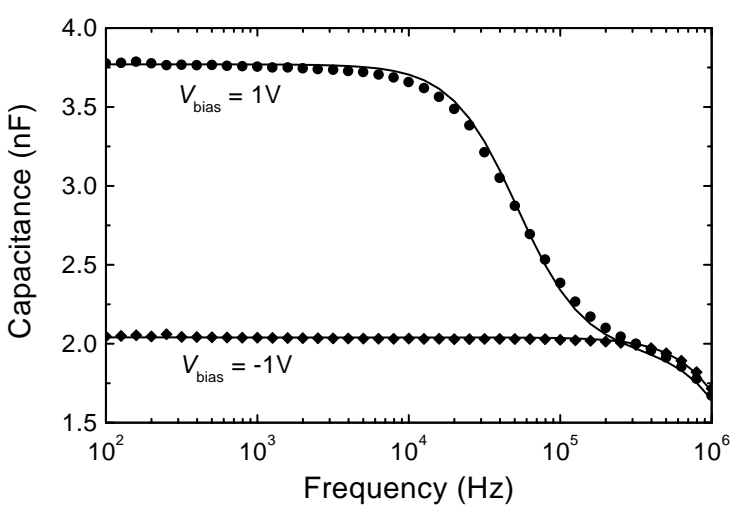

FIG. 27. Frequency dependent capacitance of an ITO/NPB/Alq/Ca device with 60nm NPB and 60nm Alq for two different values of the applied bias, together with fit curves using the equivalent circuit shown in Fig. 26 and an additional series resistance for the leads. 
(a)

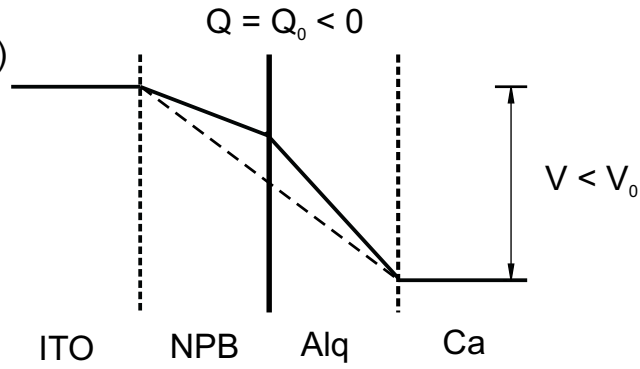

(b)

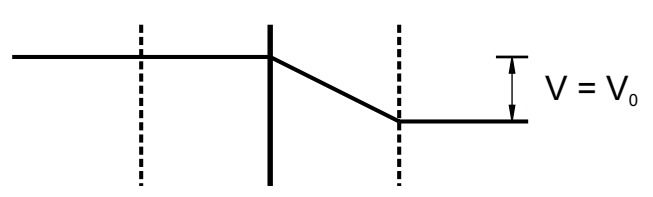

(c)

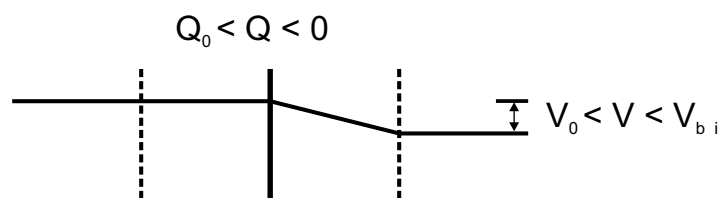

(d)

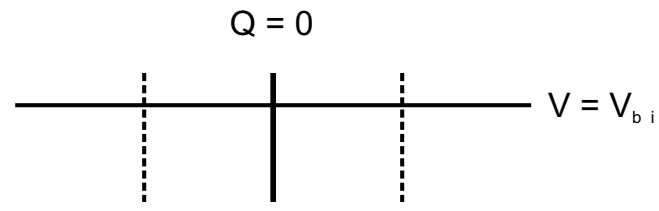

(e)

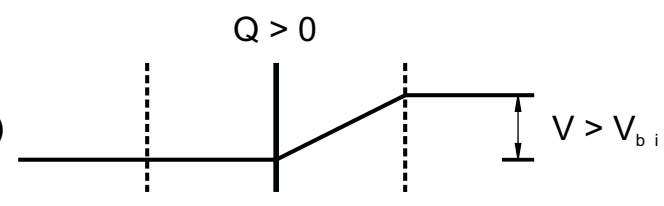

FIG. 28. Spatial dependence of the electrical potential inside an ITO/NPB/Alq/Ca device under different bias conditions: (a) For large reverse bias the interfacial charge $Q_{0}$ creates a jump of the electrical field, which is equivalent to the change of slope of the potential at the interface. The dashed line indicates the situation without interfacial charge. (b) At $V=V_{0}$ the flat band condition is reached in NPB. (c) For $V_{0}<V<V_{b i}$ NPB stays in the flat band condition, the amount of negative charge at the interface becomes smaller and concomitantly the jump of the electric field is reduced. (d) At $V=V_{b i}$ the flat band condition in both layers is achieved and the interfacial charges are fully neutralized. (e) For $V>V_{b i}$ injected positive carriers accumulate at the interface to generate again a jump of the electric field. 